\title{
Post-Weaning Social Isolation of Rats Leads to Long-term Disruption of the Gut Microbiota-Immune-Brain Axis
}

\footnotetext{
*Fionn Dunphy Doherty ${ }^{1}$, *Siobhain M O’Mahony ${ }^{2,3}$, Veronica L Peterson ${ }^{3}$, Orla O’Sullivan ${ }^{3,4}$, Fiona Crispie ${ }^{4}$, Paul D Cotter ${ }^{3,4}$, Peter Wigmore ${ }^{1}$, Madeleine V King ${ }^{1}$, "John F Cryan ${ }^{2,3}$, "Kevin CF Fone ${ }^{1}$
}

${ }^{1}$ School of Life Sciences, Medical School, Queen's Medical Centre, The University of Nottingham, Nottingham, United Kingdom NG7 2UH

${ }^{2}$ Department of Anatomy and Neuroscience, University College Cork, Ireland ${ }^{3}$ APC Microbiome Institute, University College Cork, Ireland

${ }^{4}$ Teagasc Food Research Centre, Moorepark, Fermoy, Ireland

\author{
*Joint First authors \\ \#oint Senior Authors \\ Correspondence: kevin.fone@nottingham.ac.uk
}

Tel: $\quad+441158230131$

Fax: $\quad+441158230142$

Keywords: isolation rearing, microbiota, cytokines, anxiety, learning and memory, schizophrenia, neurogenesis

Abbreviations: ANOVA, analysis of variance; BrdU, 5-bromo-2'-deoxyuridine; CFR, conditioned freezing response; GH, Group-housed; HPA, hypothalamic pituitary-adrenal axis; NeuN, neuronal nuclei; NOD, novel object discrimination; PFC, prefrontal cortex; SI, social isolation; SEM, standard error of the mean. 


\section{Abstract}

Early-life stress is an established risk for the development of psychiatric disorders. Postweaning isolation rearing of rats produces lasting developmental changes in behavior and brain function that may have translational pathophysiological relevance to alterations seen in schizophrenia, but the underlying mechanisms are unclear. Accumulating evidence supports the premise that gut microbiota influence brain development and function by affecting inflammatory mediators, the hypothalamic-pituitary-adrenal axis and neurotransmission, but there is little knowledge of whether the microbiota-gut-brain axis might contribute to the development of schizophrenia-related behaviors. To this end the effects of social isolation (SI; a well-validated animal model for schizophrenia)-induced changes in rat behavior were correlated with alterations in gut microbiota, hippocampal neurogenesis and brain cytokine levels. Twenty-four male Lister hooded rats were housed in social groups (group-housed, GH, 3 littermates per cage) or alone (SI) from weaning (post-natal day 24) for four weeks before recording open field exploration, locomotor activity/novel object discrimination (NOD), elevated plus maze, conditioned freezing response (CFR) and restraint stress at one week intervals. Post-mortem caecal microbiota composition, cortical and hippocampal cytokines and neurogenesis were correlated to indices of behavioral changes. SI rats were hyperactive in the open field and locomotor activity chambers traveling further than $\mathrm{GH}$ controls in the less aversive peripheral zone. While SI rats showed few alterations in plus maze or NOD they froze for significantly less time than GH following conditioning in the CFR paradigm, consistent with impaired associative learning and memory. SI rats had significantly fewer BrdU/NeuN positive cells in the dentate gyrus than $\mathrm{GH}$ controls. SI rats had altered microbiota composition with increases in Actinobacteria and decreases in the class Clostridia compared to $\mathrm{GH}$ controls. Differences were also noted at genus level. Positive correlations were seen between microbiota, hippocampal IL-6 and IL-10, conditioned freezing and open field exploration. Adverse early-life stress resulting from continuous SI increased several indices of 'anxiety-like' behavior and impaired associative learning and memory accompanied by changes to gut microbiota, reduced hippocampal IL-6, IL-10 and neurogenesis. This study suggests that early-life stress may produce long-lasting changes in gut microbiota contributing to development of abnormal neuronal and endocrine function and behavior which could play a pivotal role in the aetiology of psychiatric illness. 


\section{Introduction}

Exposure to early-life stress is an established risk factor for the development of several common psychiatric conditions; including anxiety-related disorders, schizophrenia, posttraumatic stress disorder and depression (Heim et al., 1997). Furthermore, stress is the strongest predictor of developing depression in clinical populations (Kendler et al., 1993), and developmental rodent models of psychiatric illness often contain a stress component to attempt to provide translational relevance (Nestler and Hyman, 2010). In rodents adverse early-life events modify brain development, affecting resultant adult behavior and often producing neurobiological sequelae that resemble changes seen in depression and schizophrenia which may therefore contribute to development of these common psychiatric disorders (Lapiz et al., 2003; Pryce et al., 2005).

Social isolation of rat pups from weaning (hereafter referred to as $\mathrm{SI}$ ) is an established neurodevelopmental model producing robust behavioral, neurochemical and anatomical changes reminiscent of several core features of schizophrenia and depression (Fone and Porkess, 2008; Jones et al., 2011). These include attenuated exploratory habituation to a novel arena (Gentsch et al., 1982; Meffre et al., 2012; Sahakian et al., 1977), enhanced 'anxiety-related' behavior in the elevated plus maze (Parker and Morinan, 1986; Weiss et al., 2004) and reduced social interaction (Mclntosh et al., 2013; Watson et al., 2016; Wongwitdecha and Marsden, 1996). SI rats also show impaired learning and memory in multiple cognitive domains including; visual learning and memory (novel object discrimination) pre-attention processing (pre-pulse inhibition of acoustic startle) and associative learning (conditioned freezing response, CFR) (Bakshi et al., 1998; Bianchi et al., 2006; Fone and Porkess, 2008; Gaskin et al., 2016; McIntosh et al., 2013; Meffre et al., 2012). 
SI also modifies the hypothalamic pituitary-adrenal (HPA) axis stress response. However basal levels of adrenocorticotrophic hormone and corticosterone have been reported to be increased (Weiss et al., 2004) or unaltered (Scaccianoce et al., 2006; Schrijver et al., 2002), which might depend on the strain of rat (Malkesman et al., 2006) or the severity of the isolation procedure (e.g. use of sawdust bedding or housing on a grid floor) (Fone and Porkess, 2008). One group (Serra et al., 2005) reported SI caused hypersensitivity of the pituitary gland to intraventricular injection of corticotrophin-releasing hormone and impaired glucocorticoid receptor mediated negative feedback of the HPA axis, analogous to suppression of feedback inhibition seen in severe depression. Furthermore, Muchimapura et al. (2002) demonstrated that SI can affect the stress response by altering neurodevelopment of the serotonergic innervation of the hippocampus.

Despite a wealth of research, the mechanisms underlying the developmental changes produced by $\mathrm{SI}$ are not established. One possible contributory factor, which has so far received little investigation, is that isolation rearing modifies the microbiota-gut-brain axis thereby disrupting normal neural, endocrine, metabolic and immunological bicommunication between gut microbiome and the brain (Jones et al., 2006). This could then adversely affect neuronal development and adult behavior (see reviews, (Cryan and Dinan, 2012; Parashar and Udayabanu, 2016; Sarkar et al., 2016)). Indeed recent research has implicated alterations in the composition of gut microbiota as a potential factor in the aetiology of a number of central nervous system disorders; including autism (Williams et al., 2011), anxiety and depression (Foster and McVey Neufeld, 2013; Naseribafrouei et al., 2014) but there has been a paucity of research on any link between gut microbiota and schizophrenia (Dinan et al., 2014) despite association with autoimmunity and gastrointestinal disorders (Severance et al., 2016). Early studies of microbiota established that germ-free mice show increased stress 
reactivity (Sudo et al., 2004), repetitive self-grooming and reduced social interaction which are all reversed by colonization of the gut (Desbonnet et al., 2014). Conversely studies also demonstrate that exposure of rodents to a stressor alters microbiota resulting in an associated increase in pro-inflammatory cytokines (Bailey et al., 2011; Galley and Bailey, 2014).

For example, maternal separation of rat pups not only enhances anxiety-related behavior and cytokine levels but also changes gut microbiota (O'Mahony et al., 2009) and treatment with probiotics has a comparative effect to the SSRI citalopram; attenuating some of these changes (Desbonnet et al., 2010). However, there is limited data on the role of the microbiota in any animal model for schizophrenia. To this end we characterised the impact of housing rats in SI from weaning on a behavioral tasks (selected for translational relevance to core symptoms seen in schizophrenia) evaluating performance in cognitive, 'anxiety-like' and stress paradigms and correlated these with changes in gut microbiota, hippocampal and prefrontal cortical (PFC) cytokine, plasma corticosterone levels and hippocampal neurogenesis (BrdU/NeuN expressing neurones). Associations are made between changes in specific behavioral phenotypes and gut microbiota in rat littermates either housed in small social groups $(\mathrm{GH})$ or in $\mathrm{SI}$ and the potential translational relevance of these findings to common neurodevelopmental psychiatric disorders is discussed. 


\section{Methods}

\subsection{Animals}

Twenty-four male Lister hooded rats (from four equal sized litters) were obtained from Charles River UK on post-natal day 24 and weaned on arrival. Each litter was divided such that half the pups were group-housed $(\mathrm{GH})$ and the other half reared alone (SI). Rats were housed in plastic cages $(32 \times 51 \mathrm{~cm}, \mathrm{GH}$ or $25 \times 42, \mathrm{SI})$ containing sawdust bedding with metal grid lids, and had visual, auditory and olfactory contact with conspecifics in the same holding room. Rats were weighed once weekly (when bedding was changed). Food and water were available ad libitum and rats were kept on a twelve hour light/dark cycle (lights on 07.00h) at constant temperature $\left(21 \pm 2{ }^{\circ} \mathrm{C}\right)$ and humidity $(45 \pm 15 \%)$. On post-natal day 56 rats received 5-bromo2'-deoxyuridine (BrdU, a marker of cell proliferation, Sigma Aldrich, $150 \mathrm{mg} / \mathrm{kg}$, i.p.) and housed for a further week before behavioral testing began (Fig. 1). All behavioral studies were conducted between 08.00 and $17.00 \mathrm{~h}$ with $\mathrm{SI}$ and $\mathrm{GH}$ rats being analysed in a random order to minimise any temporal effect, and before each test the arena was cleaned with $20 \%$ ethanol and dried to remove odour cues. All procedures were carried out in accordance with the UK Animals (Scientific Procedures) Act (1986), with approval of the University of Nottingham Local Ethical Review Committee, and conformed to the ARRIVE guidelines (Kilkenny et al., 2010; McGrath et al., 2010). Behavioral studies were conducted during the light phase of the circadian cycle to enable comparison with all our previous work with this model and the majority of other publications. Although there may be sex differences in behavioral and neuroendocrine responses following $\mathrm{SI}$, this was not the primary focus of the current studies which were therefore performed on male rats to minimise the number of animal required according to the $3 R^{\prime}$ s principle. 


\subsection{Open Field Behavior}

As an ethological index of anxiety, behavior was recorded in a novel circular open field (on post-natal day 59, Fig. 1.). Rats were placed in the centre of the unfamiliar arena $(75 \mathrm{~cm}$ diameter with $45 \mathrm{~cm}$ high walls, lined with grey card) at $120 \mathrm{Lux}$ for $15 \mathrm{~min}$ and behavior recorded using computerised tracking (Noldus, Ethovision XT ver7.1). Ambulatory speed and distance travelled in the peripheral and central zone $15 \mathrm{~cm}$ from the wall defined on Ethovision) together with time spent rearing and body grooming and the number of faecal boli were measured as indices of 'anxiety levels'.

\subsection{Spontaneous locomotor activity in a novel cage}

Isolation rearing-induced hyperactivity in response to a novel environment was measured to confirm development of the isolation syndrome and as a further index of neophobia as described previously (Gaskin et al., 2016; King et al., 2009; Watson et al., 2016) on post-natal day 65 (Fig. 1). Each rat was placed in an unfamiliar perspex arena $(39 \mathrm{~cm} \times 23.5 \mathrm{~cm} \times 24.5 \mathrm{~cm}$ for $1 \mathrm{~h}$ ) to record horizontal locomotor activity and rearing using a computerised infra-red activity system (Photobeam Activity System, PAS).

\subsection{Novel object discrimination (NOD)}

Twenty four hours following locomotor assessment (and consequent habituation to the arena) novel object discrimination (NOD) was assessed to evaluate visual recognition memory (Lyon et al., 2012) as described before (Jones et al., 2011; Millan et al., 2010). In turn, each rat was tested for NOD using the following sequence. Each rat was re-acclimatised to the same perspex arena previously used for locomotor activity for $3 \mathrm{~min}$, immediately placed back in the home cage for $1 \mathrm{~min}$ and then returned to the arena to receive two consecutive $3 \mathrm{~min}$ 
object exploration trials separated by a $2 \mathrm{~h}$ inter-trial interval in the home cage. During the first (familiarisation) trial, two identical white bottles $(8 \mathrm{~cm}$ high, $5 \mathrm{~cm}$ diameter) were placed in opposite corners of the arena ( $5 \mathrm{~cm}$ from the side and $10 \mathrm{~cm}$ from the end wall). During the second (choice) trial, one object (chosen in a pseudo-random balanced design) was replaced with a novel unfamiliar object (white bottle with black horizontal stripes). During each trial the time spent exploring each object (sniffing or active vibrissae with the nose within $1 \mathrm{~cm}$ of the object) was recorded by stop watches. Climbing on or chewing at the object was not counted as exploration. The discrimination ratio (novel/(novel + familiar times)) was derived from the raw data to further determine object preference.

\subsection{Elevated plus maze}

Exploration of the elevated plus maze was measured to evaluate any change in 'anxiety-like behavior' associated with isolation rearing, as described previously by us (Bull et al., 2004; Fone et al., 1996). Each rat was placed in the centre of the maze facing a closed arm and left to explore for $5 \mathrm{~min}$. The $70 \mathrm{~cm}$ high black perspex maze (with foam mats beneath to prevent injury from falls) consisted of four $45 \mathrm{~cm}$ long arms, two opposing arms being open (no walls) and two enclosed by $10 \mathrm{~cm}$ high walls. Each arm was divided into equal dimension outer and inner zones and behavior monitored using software (Noldus Ethovision XT ver7.1) to determine time and entries into each arm and zone and distance travelled. In addition the number of unprotected head-dips (head-dip below the floor of the open arm) and stretch attend postures (extending two front feet into the centre of an open arm without immediately entering; a risk assessment behavior) were recorded manually from video recordings. The percentage time and entries in the open arms (open/(open+closed) was calculated from the raw data, as a further index of anxiety. 


\subsection{Conditioned freezing response (CFR)}

To determine any change in associative learning and memory, a two compartment Geller box was used to record CFR behavior, a reliable index of associative learning, memory and extinction, as described previously (Jones et al., 2011; Woods et al., 2012). On the conditioning day (post-natal day 79, Fig. 1) each rat was placed in the light side of a computerised shuttle box $(24.8 \times 25.2 \times 24.3 \mathrm{~cm}$, Panlab) for 30 s before the compartment door temporarily opened to allow transition to the dark side and latency to enter was recorded. Thirty seconds later a conditioned stimulus ( 5 s light and $3 \mathrm{~Hz}, 89 \mathrm{~dB}$ tone) signalled the start of an unavoidable foot shock (unconditioned stimulus, $1 \mathrm{~s}, 0.4 \mathrm{~mA}$ during the last $1 \mathrm{~s}$ of the light and tone). Each rat received three shocks separated by 59s. Freezing behavior (in a hunched position with inactive vibrissae and totally immobile except for respiratory movement) was measured after the first two shocks. Twenty four and $48 \mathrm{~h}$ after conditioning rats were returned to the dark side of the shuttle box for 300 s, without further shocks, and time spent freezing recorded again. Immediately after the $48 \mathrm{~h}$ trial the light and tone were presented again without the foot shock and freezing recorded during the next 300 s.

\subsection{Restraint Stress}

Exposure to restraint stress has been regularly used as a psychological stress to evaluate HPA axis function (Hesketh et al., 2005) which may be modified by previous exposure to early-life SI (Fone et al., 1996). To elicit this response rats were restrained in a perspex tube ( $27 \times 7 \times$ $7 \mathrm{~cm}$ ) for $1 \mathrm{~h}$ on post-natal day 86 or 87 (Fig .1).

\subsection{Post-Mortem}


Immediately following restraint rats were humanely killed by concussion followed by immediate exsanguination and mixed arterio-venous blood was collected, centrifuged $\left(13,000 \mathrm{G}, 4^{\circ} \mathrm{C}, 5 \mathrm{~min}\right)$ and plasma decanted and frozen at $-80^{\circ} \mathrm{C}$ for plasma corticosterone determination (in duplicate from the linear portion of the curve, $20-80 \%$ maximal binding region) by ELISA (Enzo Life Sciences). The brain was hemisected to remove the whole hippocampus and prefrontal cortex (PFC) from the left side and snap frozen in liquid $\mathrm{N}_{2}$ for measurement of cytokines. The other half of the brain was placed in $4 \%$ paraformaldehyde for $24 \mathrm{~h}$ then into $30 \%$ sucrose overnight and flash-frozen $24 \mathrm{~h}$ later for immunohistochemical analysis. Caecal samples were collected under aseptic conditions for microbiota analysis.

\subsection{Mircobiota analysis}

Caecal samples were prepared for 165 sequencing on the Illumina Miseq system essentially as described in the Illumina $16 \mathrm{~S}$ library preparation workflow. In brief, the V3 and V4 region of the 16S bacterial rRNA gene were amplified using 16S primers (Sigma Aldrich Ireland Itd., Wicklow, Ireland). Kapa Robust was used to amplify the products and the number of cycles used was 30. The resulting PCR products were cleaned up using Ampure XP beads (Labplan Itd., Co. Kildare, Ireland). Dual indices and Illumina sequencing adapters (Nextera XT Index Kit, Illumina, Inc., San Diego, California, USA) were attached to the amplicons. Following another clean-up step amplicons were quantified, normalized and pooled. The resulting pool was once again cleaned before being run at the Teagasc sequencing facility on the Illumina Miseq platform.

\subsection{Bioinformatic Analysis}

Resulting FastQ forward and reverse reads were joined together using FLASH. Sequences were clustered into Operational Taxonomic Units (OTU's) and chimeras removed using USEARCH (Version 7.0-64bit). Taxonomy was assigned to OTUs with BLAST against 
Silva123.Alpha and beta diversities were calculated within Qiime (v1.9.0). PCoA plots were visualised with Emperor.

\subsection{BrdU/NeuN Immunohistochemistry}

Dual-staining immunohistochemistry was performed to identify co-existence of BrdU, a marker of cell proliferation, with the neuronal specific nuclear protein, neuronal nuclei (NeuN) in hippocampal slices. Sixty micron microtome sections were sequentially washed with phosphate buffered saline, incubated $\left(2 \mathrm{M} \mathrm{HCl}, 37^{\circ} \mathrm{C}, 30 \mathrm{~min}\right)$, washed $(5 \mathrm{~min} 0.1 \mathrm{M}$ borate buffer), incubated for $1 \mathrm{~h}$ in a $2 \%$ blocking solution (goat serum, $1 \%$ BSA containing $0.3 \%$ Triton-x100) and then primary antibody (rat anti-BrdU, 1:100) overnight at $4^{\circ} \mathrm{C}$. The next day sections were washed and incubated with secondary antibody (Alexa Flour 568 goat anti-rat, 1:500 $1 \mathrm{~h}$ room temperature). Following a further wash sections were incubated with Mouse anti-NeuN, (1:100 at $4^{\circ} \mathrm{C}$ overnight), washed again and incubated with secondary antibody (Alexa Flour 488 rabbit anti-mouse, 1:500 for $1 \mathrm{~h}$ ). Sections were mounted on gelatinised slides, rinsed with distilled water, DAPI applied (1:1000, 30s in darkness), cover slipped and positive cells quantified by an observer who was unaware of the rat housing condition.

\subsection{Cytokine and mTOR analysis.}

Hippocampal and frontal cortex samples were removed from $-80^{\circ} \mathrm{C}$ and homogenised by sonication in RIPA buffer (1ml per $12 \mathrm{mg}$ of tissue containing 1:100 protease inhibitor, P8340, Sigma). Lowry assay was performed to prepare all samples to a common protein concentration. Cytokine (IL-1 $\beta, \mathrm{IL}-6, \mathrm{IL}-10$ and TNF- $\alpha$ ) and mTOR (total and phosphorylated) levels in brain samples were determined in duplicate using a multiplex kit (MILLIPLEX MAP, Merck Millipore, UK) against standards with quality controls as described previously (Hind et al., 2016). 


\subsection{Statistical analysis}

GraphPad Prism v6 (GraphPad Software Inc., La Jolla, CA, USA) and SPSS22 (IBM Analytics, UK) were used for statistical analyses. All data were checked for normality and homogeneity of variance using Shapiro-Wilk's and Levene's tests, respectively. Locomotor activity and NOD data were analysed by Two-way repeated measures analysis of variance (RM ANOVA, with rearing condition as a between-subject factor, and time and object as repeated measures). Measures made without a time course in open field, elevated plus maze and NOD, such as choice trial discrimination ratio and cytokine, mTOR and BrdU were analysed by ANOVA (with rearing condition as a factor). In each case, where appropriate, multiple comparison post-hoc tests were used where ANOVA suggested statistical significance $(p<0.05)$ and data are expressed as mean \pm SEM. Microbiota analysis was performed in R (version 3.3.2). The vegan package (version 2.3-4) was used for alpha diversity indices and Adonis test. Differences in alpha diversity and taxonomic composition were calculated with Mann-Whitney $U$ test. Spearman correlations were performed between relative microbiota abundance and behavior and cytokine measures. All post-hoc tests and Spearman correlations included correction for multiple comparison.

\section{Results}

\subsection{Open field behavior}

SI rats travelled significantly further than $\mathrm{GH}$ control rats $(p<0.001, \mathrm{Fig} .2 \mathrm{~A})$ and at a higher overall velocity $(p<0.001$, Fig. $2 B$ ) in the whole open field thus demonstrating hyperactivity in a novel arena consistent with development of the isolation-reared behavioral syndrome. 
As expected both $\mathrm{GH}$ and $\mathrm{SI}$ rats spent significantly more time in the outer than the central (more aversive) zone of the open field (ANOVA; main effect of zone $p<0.0001$, Fig. $2 \mathrm{C}$ ) but this was unaffected by rearing condition. Further ethological analysis failed to show any significant difference in either the number of rears or time spent grooming between GH and SI rats (data not shown). In contrast, at the end of the trial SI rats had produced significantly fewer $(p<0.05)$ faecal boli than GH littermates (1.6 \pm 0.8 and $3.9 \pm 0.7$, respectively, Fig. $2 \mathrm{D})$, consistent with the development of an anxiogenic profile by SI.

\subsection{Effects of isolation-rearing on exploration and locomotor activity}

In accordance with open field data SI rats displayed significantly higher ( $p<0.01$, Fig. 2E) total horizontal locomotor activity over $1 \mathrm{~h}$ in the activity boxes compared to $\mathrm{GH}$ rats. Temporal analysis of this locomotion (Fig. 2F) showed that the change was due to a slower rate of habituation, especially during the first 30min, consistent with $\mathrm{SI}$ inducing neophobia. Although there was no significant difference in the overall total number of rears, SI rats also showed significantly more $(p<0.05)$ rears during the second $5 \mathrm{~min}$ in the box and a similar trend for reduced attenuation of rearing behavior during the first $30 \mathrm{~min}$ as seen with ambulation (data not shown).

\subsection{Novel Object Discrimination}

During the first (familiarisation) trial of the NOD task neither SI nor GH controls showed any preference for either identical object (Table 1). Analysis of differential object exploration in the second (choice) trial (Table 1) showed that both SI and GH rats tended to spend more time exploring the novel object, but this did not reach significance (ANOVA showed no main

effect of housing $\left(F_{(1,21)}=2.32, P=0.14\right)$ or object $\left.\left(F_{(1,21)}=2.35, P=0.14\right)\right)$. Neither did the 
apparent small reduction in discrimination ratio in the $\mathrm{SI}$ rats reach significance.

\subsection{Elevated Plus Maze}

The effect of post-weaning SI was examined on a task widely used to assess unconditioned 'anxiety-like' behavior; the elevated plus maze (Fone et al., 1996; Griebel et al., 1997). Irrespective of housing, rats spent significantly longer $(p<0.001)$ in the closed $(G H, 61.5 \pm 2.7$; $\mathrm{SI}, 59.4 \pm 3.3 \mathrm{~s})$ than the open $(\mathrm{GH}, 38.5 \pm 2.7 ; \mathrm{SI}, 40.6 \pm 3.3 \mathrm{~s})$ arms of the maze. ANOVA showed a main effect of arm type (Open vs Closed; $F_{(1,44)}=47.89, p<0.001$ ) but no main effect of housing $\left(F_{(1,44)}=0.00001, p=0.99\right)$ on the pattern of exploration of the two arm types. A very similar profile was seen for both percentage open and closed arm time and entries; ANOVA showed a significant main effect of arm type $\left(F_{(1,44)}=47.89, p<0.001\right.$ and $F_{(1,44)}=12.57$, $p<0.001$, respectively) but no main effect of housing during development and no significant arm type $\mathrm{x}$ housing interaction for either parameter. Thus rats spent significantly more time in, and made more entries into, the closed than the open arms irrespective of housing during development. There was no significant difference in distance travelled by GH or SI rats $(2.43 \pm 0.10$ and $2.69 \pm 0.10 \mathrm{~m}$ respectively, not significant Student's $t$-test $)$ on the plus maze, confirming that difference in ambulation did not confound interpretation of ethological measures made as indices of anxiety in this task. More detailed ethological analysis of behaviors failed to show any significant effect of housing condition on unprotected stretch attend postures (risk assessment) or open arm head-dips (directed exploratory behavior; data not shown).

\subsection{Conditioned Freezing Response}


To assess the effect of post-weaning SI on associative learning and memory, freezing behavior was measured as an index of cognition in a cue and context motivated fear conditioning paradigm (Jones et al., 2011; Woods et al., 2012).

During the acquisition phase SI rats froze for significantly less time $(p<0.001$ Sidak's post-hoc test) than $\mathrm{GH}$ rats after the first shock, but both housing groups froze for the same duration after the second shock such that there was a main effect of housing $\left(F_{(1,22)}=6.97, p<0.05\right.$, Fig. 3A). This suggests that although isolates have a different initial response to the first foot shock they then find the conditioning equally aversive (Fig. 3A).

At both 24 and $48 \mathrm{~h}$ after conditioning GH froze for significantly longer $(p<0.05)$ than SI rats when returned to the conditioning chamber without exposure to any further shock or cue, consistent with impaired contextual learning and memory. SI also froze for significantly less time $(p<0.01)$ than $\mathrm{GH}$ rats at $48 \mathrm{~h}$ post-conditioning when the light and tone (but no shock) was re-applied demonstrating impaired cue-mediated learning and memory. ANOVA confirmed there was a significant main effect of housing $\left(F_{(1,22)}=12.63, p<0.01\right)$ on freezing duration across all three trial periods (Fig. 3B).

\subsection{Restraint stress-induced plasma corticosterone response}

Restraint caused the expected elevation in plasma corticosterone (from $69.0 \pm 3.6$ to $92.5 \pm$ 3.6 in $\mathrm{GH}$ and $70.4 \pm 4.9$ to $94.0 \pm 7.6 \mathrm{ng} / \mathrm{ml}$ in $\mathrm{SI}$ rats) but this not modified by housing condition during development. Thus two-way ANOVA showed no main effect of housing $\left(F_{(1,}\right.$ $20)=0.084, p=0.77)$ but a main effect of restraint $\left(F_{(1,20)}=20.68, p<0.001\right)$ on plasma corticosterone.

\subsection{Hippocampal cell proliferation and neurogenesis}


The level of neuronal cell proliferation as an index of neurogenesis in the dentate gyrus of the hippocampus was examined using BrdU/NeuN dual-labelled immunohistochemistry (see representative image Fig. 4A). SI showed a significant reduction ( $p<0.05$, Student's $t$-test) in cells in the dentate gyrus dual-labelled for BrdU and NeuN compared to GH rats (172 \pm 19 and $243 \pm 23$, respectively) showing that isolation rearing attenuated the number of cells proliferating into a neuronal phenotype during the study (Fig. 4B).

\subsection{Hippocampal and prefrontal cortical (PFC) cytokine and mTOR levels}

To determine any change in markers of pro- and anti-inflammatory cytokines, IL-1 $\beta$, IL-6, IL10 and TNF- $\alpha$ levels were measured in hippocampal and PFC extracts using multiplex assays.

As rats received either restraint or were housed in the home cage (control) for the hour immediately before tissue collection, and short term physical restraint can alter brain cytokine levels, 2-way ANOVA with housing and restraint as factors was performed on cytokine levels. In the PFC there was no significant main effect of restraint, nor any housing restraint interaction on any of the cytokines measured and only IL- 6 in the hippocampus showed a housing $\mathrm{x}$ restraint interaction, so results for each cytokine were pooled to determine the impact of housing in Table 2. Of particular note for data pooled irrespective of restraint, significant reductions $(p<0.05)$ in both IL-6 and IL-10 (Table 2 ) occurred in the hippocampus, but not the PFC of SI compared to control GH littermates. In contrast, no significant alterations in IL-1 $\beta$ or TNF $\alpha$ occurred in either brain region (Table 2). Further analysis of hippocampal IL-6 (Fig. 5B) by 2-way ANOVA showed a significant interaction $\left(F_{(1,19)}=11, p<0.05\right)$ between housing and restraint, such that hippocampal IL-6 was significantly lower in SI rats that had been restrained than either SI home cage $(P<0.05)$ or $\mathrm{GH}$ restrained rats $(\mathrm{P}<0.05)$. In comparison no similar significant interaction was seen with hippocampal IL-1 $\beta$ (Fig. 5A) or other cytokines (data not shown). 
Although there were no significant differences in levels of phospho-mTOR or total mTOR in either brain region according to housing condition, there was a small but significant increase in the ratio of phospho-mTOR to total mTOR in the PFC $(p<0.05)$ which was not observed in the hippocampus ( $p=0.78)$. This suggests that selective enhancement of the mTOR signalling pathway might occur in the PFC as a consequence of SI (Table 3).

\subsection{Microbiota composition and analysis}

At the phylum level, Mann-Whitney test revealed significant differences in low relative abundance ( $1 \%)$ Actinobacteria $(p=0.04)$ with an increase in SI compared to GH rats (Fig. 6 and Table 4). Increases in phylum Actinobacteria were attributed to increases in genus Rhodococcus ( $p=0.03$; Fig. 7). SI significantly decreased Clostridia $(p=0.03$ ) at the class level; this was reflected in decreases in order Clostridiales $(p=0.03)$, family Clostridiaceae group 1 $(p=0.01)$ and Peptostreptococcaceae ( $p=0.02$; Fig. 8). However, at the genus level both increases and decreases were observed within the Clostridia class, with increases in Defluvitaleaceae UCG-011 $(p=0.03)$, Eubacterium oxidoreducens group $(p=0.02)$, Marvinbryantia $(p=0.04)$, and decreases in Lachnospiraceae UCG-009 ( $p=0.02)$, Ocillospira $(p=0.03)$ and Papillibacter $(p=0.02)$. Mann-Whitney test revealed significant increases in other genra of bacteria in SI compared to $\mathrm{GH}$, including Bacillus ( $p=0.02$ ), Prevotellaceae UCG-001 $(p=0.04)$, and Veillonella $(p=0.03 ;$ Table 4). Alpha diversity analysis showed a trend toward decreased diversity in the SI compared to GH rats, but this was not significant (Shannon index, $\mathrm{p}=0.28$ ). Beta diversity analysis by group showed a trend toward clustering by group, however this trend was also not significant (Adonis, $\mathrm{p}=0.12$ ). 
Correlations were performed between salient individual representative values of specific behaviors and neurochemical measures that had been significantly altered by housing and significant changes in microbiota found in the same rat, thus focussing on those parameters that were significantly altered by early-life housing. Microbiota that significantly differed between groups were highly correlated to hippocampal IL-6 and faecal boli in the open field test (Fig. 9). Taxa in the order Clostridales were positively correlated to faecal boli $(\rho=0.54$, $p=0.01$ ) in all groups. This positive correlation continued at the family level (Ruminococcaceae, $\rho=0.57, p=0.01$ ), and genus level (Papillibacter, $\rho=0.57, p=0.01$; Oscillospira, $\rho=0.47, p=0.02$ ). Many of the same taxa in the order of Clostridales were negatively correlated to distance travelled $(\rho=-0.52, p=0.02)$ and velocity $(\rho=-0.52, p=0.02)$ in the open field test. Relative abundance of bacteria in the order Bacillales was negatively correlated to faecal boli $(\rho=-0.44, p=0.03)$ and hippocampal IL-6 $(\rho=-0.53, p=0.01)$, with significance remaining at the Family and Genus levels. Bacteria in the order Bacillales was also positively correlated to distance travelled $(\rho=0.59, p=0.01)$ and velocity $(\rho=0.59, p=0.01)$ in the open field, significant correlations remained at the family and genus level. Further correlations could be seen in microbiota and other measures significantly different between groups. Hippocampal IL-10 $(\rho=0.56, p=0.02)$ and IL-6 $(\rho=0.48, p=0.02)$ was positively correlated to Peptostreptococcaceae. Negative correlations to $48 \mathrm{~h}$ pre-cue measures from the CFR task were seen in genra Veillonella $(\rho=-0.45, p=0.03)$ from order Selenomonadales and Eubacterium oxidoreducens group $(\rho=-0.41, p=0.05)$ belonging to the order Clostridales. Negative correlations were also seen in genus Defluviitaleaceae UGC-001 of the order Clostridales for CFR freezing time at $24 \mathrm{~h}(\rho=-0.50, p=0.02)$ and $48 \mathrm{~h}$ post-conditioning $(\rho=-0.54$, $\mathrm{p}=0.01)$. 


\section{Discussion}

The current study demonstrates that rearing rats in SI from weaning produced several behavioral changes akin to those seen in disorders such as schizophrenia and depression, including cognitive impairment, reduced neurogenesis (decreased DG BrdU/NeuN positive cells) and changes in hippocampal cytokine levels and of particular note is the first to show accompanying alterations in microbiota composition. As expected rearing rats in SI from the point of weaning altered horizontal activity both in the open field and locomotor activity task; SI rats being hyperactive is a reliable characteristic feature of the resultant isolation syndrome' observed in almost all previous studies (Fone and Porkess, 2008; Gentsch et al., 1981; King et al., 2009; McIntosh et al., 2013; Watson et al., 2016). This increased activity in a novel environment is consistent with SI rats demonstrating neophobia (Gentsch et al., 1988; Sahakian et al., 1977). SI-induced hyperactivity is accompanied by increased spontaneous activity in (Fabricius et al., 2010) and enhanced dopamine release from (Robbins et al., 1996) ventral tegmental dopamine neurones, which is attenuated by antipsychotic drugs (McIntosh et al., 2013), and thus regarded as having face validity to the positive symptoms of schizophrenia (van den Buuse, 2010). In contrast, SI rats showed no overt changes compared to GH littermates in the elevated plus maze. This is contrary to several previous studies where SI rats show reduced total arm and percentage open entries respectively (Parker and Morinan, 1986; Weiss et al., 2004). This study also found no differences in anxiety-related behaviors in the open field (rearing and grooming) although both groups spent significantly more time in the less aversive outer zone and more time in the closed arms of the plus maze as expected. Interestingly SI rats left significantly fewer faecal boli in the open field consistent with stress-induced activation of the sympathetic nervous system which reduces defecation 
(Perhach and Barry, 1970), although studies have found other early-life stressors such as maternal separation increased faecal boli accompanying adult exposure to a novel stress (O'Mahony et al., 2009).

A variety of cognitive deficits frequently accompany psychiatric illness so rats were assessed for visual and associative learning and memory using the NOD and CFR tasks, respectively. In the NOD task, although there was a tendency for SI rats to have a lower discrimination index they did not show the expected impairment in visual discrimination (Bianchi et al., 2006; Gaskin et al., 2016; Mclntosh et al., 2013; Watson et al., 2016) possibly because of high interindividual variation in this cohort. Although SI rats froze less than group-hosed littermates after the first conditioning shock in the CFR chamber they froze for the same duration after the second shock, suggesting both groups found the stimulus aversive and that impaired acquisition did not contribute to altered conditioned freezing behaviour but it is difficult to exclude this possibility. In agreement with several previous studies (McIntosh et al., 2013; Watson et al., 2016) SI rats showed decrease freezing on re-exposure to both the conditioning context and cue in the CFR paradigm indicative of impairment in associative learning and memory following this early-life stress. Consistent with our previous observation SI rats showed an elevation of the ratio of active (phospho-mTOR) to inactive mTOR in the PFC which we have previously shown to be associated with cognitive impairment (Meffre et al., 2012). Compared with home-cage housed rats, plasma corticosterone levels were significantly elevated $1 \mathrm{~h}$ after restraint but to a comparable level in $\mathrm{SI}$ and $\mathrm{GH}$ littermates, thus irrespective of housing during development. Previous research has shown that exposure to a variety of early-life stressors including maternal separation or SI can modify the HPA axis and amplify the plasma corticosterone response to exposure to an acute stress (O'Mahony et al., 2009; Plotsky et al., 2005). As no time course assessment of the corticosterone response was 
available in the current study it is possible that plasma corticosterone had reached a ceiling level following this period of restraint which would mask any housing effect. The impact of SI on corticosterone levels is varied and probably depends on the stressor used as well as the temporal relationship of the measure to stress application (Scaccianoce et al., 2006; Serra et al., 2005; Weiss et al., 2004).

Adult neurogenesis occurs in the subgranular zone of the hippocampal dentate gyrus in rats (Kuhn et al., 1996) and dysfunction of this process is implicated in several human psychiatric disorders. Of note neurogenesis is reduced by stress and increased by antidepressants (Duman et al., 1999; Levone et al., 2015; Malberg et al., 2000). Consistent with this regulation and previous studies using rodent models of early-life stress (Mirescu and Gould, 2006) SI rats showed a significant reduction in BrdU/NeuN dual-positive dentate gyrus neurons. As changes in neurogenesis have been associated with cognitive deficits (Rola et al., 2004) and proposed to occur in depression and schizophrenia (Millan et al., 2012) their contribution to change in hippocampal dependent cognitive function, such as impaired CFR reported here, is clearly of great interest. Moreover, gut microbiota is associated with changes in hippocampal neurogenesis; a lack of microbiota in germ-free mice being associated with increased neurogenesis (Ogbonnaya et al., 2015). Conversely, antibiotic and probiotic-induced changes in the microbiota are also associated with alterations in neurogenesis (Mohle et al., 2016). Furthermore, adult germ-free mice have enlarged hippocampi and shorter less branched ventral hippocampal pyramidal neurons (consistent with dendritic atrophy) which might contribute to concomitant changes in anxiety and cognition (Jiang et al., 2015), potentially resulting from alteration in gut microbiota during development.

Alterations in the immune system are implicated in the pathophysiology of Major Depressive Disorder and also cardinal to the brain-gut axis interaction with the immune system playing a 
major role in the communication between the gut microbiota and the brain (Dinan and Cryan, 2017; O'Mahony et al., 2017). Previous research has shown that maternal separation results in an enduring pro-inflammatory phenotype in rats (O'Mahony et al., 2009) but few studies have examined changes following adolescent SI. Ko et al. (2015) found elevated plasma levels of the pro-inflammatory cytokines (IL-1 $\beta$, IL- 6 and TNF $\alpha$ ) after four weeks isolation rearing of Sprague-Dawley rats but there are no reports of changes in brain regions as documented herein. Of interest, the primary changes in cytokine levels found in this study were restricted to the hippocampus (significant reductions in IL-10 and IL-6 in SI rats) and not replicated in the PFC, implying brain regional specificity that might contribute to hippocampal-dependent phenotypic changes in cognitive behavior and/or neurogenesis (Hueston et al., 2017). A reduction in the anti-inflammatory cytokine IL-10 is consistent with findings from the microbiota levels in the current study and previous research (Scarpa et al., 2011). However, the decrease in IL-6 is unexpected since, at least in the periphery, elevated IL-6 has been associated with a depressive-like phenotype and alterations in the microbiome in mice (Zhang et al., 2017). Hippocampal IL-1 $\beta$ has been shown to be elevated by a variety of acute stressors, such as lipopolysaccharide injection or inescapable footshock, however this rise is prevented by a simultaneous rapid rise in corticosterone (Nguyen et al., 1998) or following repeated exposure to stress (Lovelock and Deak, 2017), consistent with the absence of IL-1 $\beta$ elevation in this study. Interestingly restraint stress of Wistar rats for 1 h elevated plasma corticosterone and hippocampal IL-6 mRNA (Chen et al., 2016) analogous to that seen herein in GH rats. In contrast, in this study SI rats that received restraint had significantly lower hippocampal IL-6 than GH-restrained or SI home cage rats suggesting that exposure to chronic early-life stress (SI) may have attenuated the usual brain cytokine response evoked when adult rats are exposed to a severe short-term physical stressor. Further studies on a larger cohort with 
sufficient statistical power specifically designed to investigate this phenomenon would be worthwhile to establish if this is reproducible, occurs with other cytokines and could represent a maladaptive response. One of the first studies to measure brain (rather than plasma or cerebrospinal fluid) cytokine levels in patients with schizophrenia (Pandey et al., 2017) reported reduced IL-10 but, converse to the current study, elevated IL-6. However as most patients were on antipsychotics and many also used of drugs of abuse which could alter cytokines and measurement was in the PFC, and not the hippocampus, this could account for the apparent translational discrepancy with this study.

Analysis of the microbiota revealed a number of differences that may contribute to phenotypic changes in SI rats mediated in part via the gut-brain axis bicommunication. Peptostreptococcaceae was lower in the SI rats and positively correlated to the lower IL-6 level in the hippocampus. Papillibacter which was decreased in SI rats shows preferential occurrence in healthy controls across all populations (Rehman et al., 2016) and may represent a potential indicator of health status. Oscillospira was significantly reduced in SI rats and a similar observation has been made in Crohn's disease (Perez-Brocal et al., 2013), potentially indicating that this bacteria is associated with immune alterations. Interestingly elevation in Actinobacteria found in the current SI rats was also one of a few phyla elevated in patients with active major depressive disorder compared to that in healthy controls (Jiang et al., 2015) but any translational relevance remains to be established. Bacteria in the order Clostridales were negatively correlated with cognitive performance in the CFR task. Of note an elevation in bacteria in the family of Lactinospiraceae and Clostridiaceae was observed to correlate with impairment of NOD produced following 7 days administration of phencyclidine (a pharmacological model for schizophrenia) to Lister hooded rats (Pyndt Jorgensen et al., 2015); an impairment which was prevented by ampicillin administration, supporting the supposition 
herein that gut microbiota can modify memory performance. The complex bidirectional interaction between gut microbiota and stress-related behavior makes it difficult to determine the cause or consequence of changes seen in SI rats, which is clearly imperative to understand the neurobiological sequelae and develop novel preventative therapies. Recently it has been demonstrated that mice exposed to chronic stress develop behavioral despair accompanied by reduced faecal Lactobacillus resulting in increased circulating kynurenine levels and that restoration of intestinal Lactobacillus improved the metabolic alterations and behavioral abnormalities (Marin et al., 2017). Similarly, treatment of mice undergoing chronic social defeat with Lactobacillus rhamnosus reduced the development of 'anxiety-related' behavior, deficits in social interaction and increase in number of $\mathrm{T}$ cells producing IL-10 (Bharwani et al., 2017). Furthermore, elevating caecal levels of Bifidobacteria by 3 weeks administration of galacto-oligosaccharides prevented the lipopolysaccharide-induced elevation in cortical IL-1 $\beta$ and 5- $\mathrm{HT}_{2 \mathrm{~A}}$ receptors and anxiety behavior in a light-dark box (Sarkar et al., 2016; Savignac et al., 2016). Such integrated studies are vital to provide mechanistic insight into the interaction of microbiota and their metabolites with the immune system and brain development and resultant long-term behavioral consequences. SI in juvenile rats may alter the microbiome (perhaps because of associated stress) and with contemporaneous or subsequent changes in HPA axis (Weiss et al., 2004) and inflammatory cytokines, produce microglial activation (Schiavone et al., 2009), reduction in BDNF, loss of parvalbumincontaining GABA interneurons and synaptic pruning in PFC and hippocampus (Bianchi et al., 2006; Gaskin et al., 2016; Harte et al., 2007; Schubert et al., 2009; Silva-Gómez, 2003), alter glutamatergic corticostriatal neurotransmission (Napolitano et al., 2014) and ventral tegmental dopaminergic firing (Fabricius et al., 2010); ultimately resulting in development of 
the isolation syndrome. Careful temporal analysis of the changes in microbiota, cytokines and behavior during development of the isolation syndrome might reveal the cascade of events. In conclusion, the current study established that rearing rats in SI from weaning induces changes to behavior, neurogenesis and the gut microbiome that can have potential implications on the gut-brain axis. Differences in cytokines in the hippocampus correlated to gut microbiota changes, opening the possibility that the gut microbiota influences immune changes in the central nervous system and behavior that may contribute to the pathophysiology of psychiatric disorders. This provides opportunities to target and modify the gut microbiota and potentially improve outcome in these life-changing disorders.

\section{Acknowledgments}

This work was supported by the FP7 Marie Curie ITN award ( $\left.r^{\prime} B I R T H\right)$ to KF, which provided the salary of FD. Rat behavioural work was undertaken by FD, MK and KF. MK collected caecal samples. PW provided guidance on measurement of neurogenesis. JC and SM designed the analysis of the microbiota study which was performed by FC and PC and resultant data analysed by OO'S and VP. KF, MK and FD produced the first draft of the manuscript and all authors contributed to production of the final version. 


\section{References}

Bailey, M.T., Dowd, S.E., Galley, J.D., Hufnagle, A.R., Allen, R.G., Lyte, M., 2011. Exposure to a social stressor alters the structure of the intestinal microbiota: implications for stressorinduced immunomodulation. Brain Behav Immun 25, 397-407.

Bakshi, V.P., Swerdlow, N.R., Braff, D.L., Geyer, M.A., 1998. Reversal of isolation rearinginduced deficits in prepulse inhibition by Seroquel and olanzapine. Biol Psychiatry 43, 436445.

Bharwani, A., Mian, M.F., Surette, M.G., Bienenstock, J., Forsythe, P., 2017. Oral treatment with Lactobacillus rhamnosus attenuates behavioural deficits and immune changes in chronic social stress. BMC Med 15, 7.

Bianchi, M., Fone, K.F.C., Azmi, N., Heidbreder, C.A., Hagan, J.J., Marsden, C.A., 2006. Isolation rearing induces recognition memory deficits accompanied by cytoskeletal alterations in rat hippocampus. Eur J Neurosci 24, 2894-2902.

Bull, E.J., Hutson, P.H., Fone, K.C.F., 2004. Decreased social behaviour following 3,4methylenedioxymethamphetamine (MDMA) is accompanied by changes in $5-\mathrm{HT} 2 \mathrm{~A}$ receptor responsivity. Neuropharmacology 46, 202-210.

Chen, H.J., Spiers, J.G., Sernia, C., Lavidis, N.A., 2016. Acute restraint stress induces specific changes in nitric oxide production and inflammatory markers in the rat hippocampus and striatum. Free Radic Biol Med 90, 219-229.

Cryan, J.F., Dinan, T.G., 2012. Mind-altering microorganisms: the impact of the gut microbiota on brain and behaviour. Nature Reviews Neuroscience 13, 701-712.

Desbonnet, L., Clarke, G., Shanahan, F., Dinan, T.G., Cryan, J.F., 2014. Microbiota is essential for social development in the mouse. Molecular Psychiatry 19, 146-148. 
Desbonnet, L., Garrett, L., Clarke, G., Kiely, B., Cryan, J.F., Dinan, T.G., 2010. Effects of the probiotic Bifidobacterium infantis in the maternal separation model of depression. Neuroscience 170, 1179-1188.

Dinan, T.G., Borre, Y.E., Cryan, J.F., 2014. Genomics of schizophrenia: time to consider the gut microbiome? Mol Psychiatry 19, 1252-1257.

Dinan, T.G., Cryan, J.F., 2017. Microbes, immunity, and behavior: psychoneuroimmunology meets the microbiome. Neuropsychopharmacology 42, 178-192.

Duman, R.S., Malberg, J., Thome, J., 1999. Neural plasticity to stress and antidepressant treatment. Biological Psychiatry 46, 1181-1191.

Fabricius, K., Helboe, L., Fink-Jensen, A., Wortwein, G., Steiniger-Brach, B., Sotty, F., 2010. Increased dopaminergic activity in socially isolated rats: an electrophysiological study. Neurosci Lett 482, 117-122.

Fone, K.C., Porkess, M.V., 2008. Behavioural and neurochemical effects of post-weaning social isolation in rodents-relevance to developmental neuropsychiatric disorders. Neurosci Biobehav Rev 32, 1087-1102.

Fone, K.C., Shalders, K., Fox, Z.D., Arthur, R., Marsden, C.A., 1996. Increased 5-HT2C receptor responsiveness occurs on rearing rats in social isolation. Psychopharmacology (Berl) 123, 346352.

Foster, J.A., McVey Neufeld, K.-A., 2013. Gut-brain axis: how the microbiome influences anxiety and depression. Trends Neurosci 36, 305-312.

Galley, J.D., Bailey, M.T., 2014. Impact of stressor exposure on the interplay between commensal microbiota and host inflammation. Gut Microbes 5, 390-396.

Gaskin, P.L., Toledo-Rodriguez, M., Alexander, S.P., Fone, K.C., 2016. Down-regulation of hippocampal genes regulating dopaminergic, GABAergic, and glutamatergic function 
following combined neonatal phencyclidine and post-weaning social isolation of rats as a neurodevelopmental model for schizophrenia. Int J Neuropsychopharmacol 19.

Gentsch, C., Lichtsteiner, M., Feer, H., 1981. Locomotor activity, defecation score and corticosterone levels during an openfield exposure: A comparison among individually and group-housed rats, and genetically selected rat lines. Physiology \& Behavior 27, 183-186.

Gentsch, C., Lichtsteiner, M., Frischknecht, H.-R., Feer, H., Siegfried, B., 1988. Isolationinduced locomotor hyperactivity and hypoalgesia in rats are prevented by handling and reversed by resocialization. Physiology \& Behavior 43, 13-16.

Gentsch, C., Lichtsteiner, M., Kraeuchi, K., Feer, H., 1982. Different reaction patterns in individually and socially reared rats during exposures to novel environments. Behavioural Brain Research 4, 45-54.

Griebel, G., Rodgers, R.J., Perrault, G., Sanger, D.J., 1997. Risk assessment behaviour: evaluation of utility in the study of 5-HT-related drugs in the rat elevated plus-maze test. Pharmacol Biochem Behav 57, 817-827.

Harte, M.K., Powell, S.B., Swerdlow, N.R., Geyer, M.A., Reynolds, G.P., 2007. Deficits in parvalbumin and calbindin immunoreactive cells in the hippocampus of isolation reared rats. Journal of Neural Transmission 114, 893-898.

Heim, C., Owens, M.J., Plotsky, P.M., Nemeroff, C.B., 1997. The role of early adverse life events in the etiology of depression and posttraumatic stress disorder. Focus on corticotropin-releasing factor. Ann N Y Acad Sci 821, 194-207.

Hesketh, S., Jessop, D.S., Hogg, S., Harbuz, M.S., 2005. Differential actions of acute and chronic citalopram on the rodent hypothalamic-pituitary-adrenal axis response to acute restraint stress. J Endocrinol 185, 373-382. 
Hind, W.H., England, T.J., O'Sullivan, S.E., 2016. Cannabidiol protects an in vitro model of the blood-brain barrier from oxygen-glucose deprivation via PPARgamma and 5-HT1A receptors. Br J Pharmacol 173, 815-825.

Hueston, C.M., Cryan, J.F., Nolan, Y.M., 2017. Stress and adolescent hippocampal neurogenesis: diet and exercise as cognitive modulators. Transl Psychiatry 7, e1081.

Jiang, H., Ling, Z., Zhang, Y., Mao, H., Ma, Z., Yin, Y., Wang, W., Tang, W., Tan, Z., Shi, J., Li, L., Ruan, B., 2015. Altered fecal microbiota composition in patients with major depressive disorder. Brain Behav Immun 48, 186-194.

Jones, C.A., Watson, D.J., Fone, K.C., 2011. Animal models of schizophrenia. Br J Pharmacol $164,1162-1194$.

Jones, M.P., Dilley, J.B., Drossman, D., Crowell, M.D., 2006. Brain-gut connections in functional GI disorders: anatomic and physiologic relationships. Neurogastroenterol Motil 18, 91-103.

Kendler, K.S., Kessler, R.C., Neale, M.C., Heath, A.C., Eaves, L.J., 1993. The prediction of major depression in women: toward an integrated etiologic model. Am J Psychiatry 150, 1139-1148. Kilkenny, C., Browne, W., Cuthill, I.C., Emerson, M., Altman, D.G., Group, N.C.R.R.G.W., 2010. Animal research: reporting in vivo experiments: the ARRIVE guidelines. Br J Pharmacol 160, 1577-1579.

King, M.V., Seeman, P., Marsden, C.A., Fone, K.C.F., 2009. Increased dopamine D2High receptors in rats reared in social isolation. Synapse 63, 476-483.

Ko, C.Y., Liu, Y.P., 2015. Isolation rearing impaired sensorimotor gating but increased proinflammatory cytokines and disrupted metabolic parameters in both sexes of rats. Psychoneuroendocrinology 55, 173-183. 
Kuhn, H.G., Dickinson-Anson, H., Gage, F.H., 1996. Neurogenesis in the dentate gyrus of the adult rat: age-related decrease of neuronal progenitor proliferation. J Neurosci 16, 20272033.

Lapiz, M.D., Fulford, A., Muchimapura, S., Mason, R., Parker, T., Marsden, C.A., 2003. Influence of postweaning social isolation in the rat on brain development, conditioned behavior, and neurotransmission. Neurosci Behav Physiol 33, 13-29.

Levone, B.R., Cryan, J.F., O'Leary, O.F., 2015. Role of adult hippocampal neurogenesis in stress resilience. Neurobiol Stress 1, 147-155.

Lovelock, D.F., Deak, T., 2017. Repeated exposure to two stressors in sequence demonstrates that corticosterone and paraventricular nucleus of the hypothalamus interleukin-1beta responses habituate independently. J Neuroendocrinol 29.

Lyon, L., Saksida, L.M., Bussey, T.J., 2012. Spontaneous object recognition and its relevance to schizophrenia: a review of findings from pharmacological, genetic, lesion and developmental rodent models. Psychopharmacology 220, 647-672.

Malberg, J.E., Eisch, A.J., Nestler, E.J., Duman, R.S., 2000. Chronic antidepressant treatment increases neurogenesis in adult rat hippocampus. J Neurosci 20, 9104-9110.

Malkesman, O., Maayan, R., Weizman, A., Weller, A., 2006. Aggressive behavior and HPA axis hormones after social isolation in adult rats of two different genetic animal models for depression. Behavioural Brain Research 175, 408-414.

Marin, I.A., Goertz, J.E., Ren, T., Rich, S.S., Onengut-Gumuscu, S., Farber, E., Wu, M., Overall, C.C., Kipnis, J., Gaultier, A., 2017. Microbiota alteration is associated with the development of stress-induced despair behavior. Sci Rep 7, 43859. 
McGrath, J.C., Drummond, G.B., McLachlan, E.M., Kilkenny, C., Wainwright, C.L., 2010. Guidelines for reporting experiments involving animals: the ARRIVE guidelines. Br J Pharmacol $160,1573-1576$.

McIntosh, A.L., Ballard, T.M., Steward, L.J., Moran, P.M., Fone, K.C.F., 2013. The atypical antipsychotic risperidone reverses the recognition memory deficits induced by post-weaning social isolation in rats. Psychopharmacology 228, 31-42.

Meffre, J., Chaumont-Dubel, S., Mannoury la Cour, C., Loiseau, F., Watson, D.J., Dekeyne, A., Seveno, M., Rivet, J.M., Gaven, F., Deleris, P., Herve, D., Fone, K.C., Bockaert, J., Millan, M.J., Marin, P., 2012. 5-HT(6) receptor recruitment of mTOR as a mechanism for perturbed cognition in schizophrenia. EMBO Mol Med 4, 1043-1056.

Millan, M.J., Agid, Y., Brüne, M., Bullmore, E.T., Carter, C.S., Clayton, N.S., Connor, R., Davis, S., Deakin, B., DeRubeis, R.J., Dubois, B., Geyer, M.A., Goodwin, G.M., Gorwood, P., Jay, T.M., Joëls, M., Mansuy, I.M., Meyer-Lindenberg, A., Murphy, D., Rolls, E., Saletu, B., Spedding, M., Sweeney, J., Whittington, M., Young, L.J., 2012. Cognitive dysfunction in psychiatric disorders: characteristics, causes and the quest for improved therapy. Nature Reviews Drug Discovery $11,141-168$.

Millan, M.J., Buccafusco, J.J., Loiseau, F., Watson, D.J., Decamp, E., Fone, K.C., ThomassonPerret, N., Hill, M., Mocaer, E., Schneider, J.S., 2010. The dopamine D3 receptor antagonist, S33138, counters cognitive impairment in a range of rodent and primate procedures. Int J Neuropsychopharmacol 13, 1035-1051.

Mirescu, C., Gould, E., 2006. Stress and adult neurogenesis. Hippocampus 16, 233-238.

Mohle, L., Mattei, D., Heimesaat, M.M., Bereswill, S., Fischer, A., Alutis, M., French, T., Hambardzumyan, D., Matzinger, P., Dunay, I.R., Wolf, S.A., 2016. Ly6C(hi) monocytes provide 
a link between antibiotic-induced changes in gut microbiota and adult hippocampal neurogenesis. Cell Rep 15, 1945-1956.

Muchimapura, S., Fulford, A.J., Mason, R., Marsden, C.A., 2002. Isolation rearing in the rat disrupts the hippocampal response to stress. Neuroscience $112,697-705$.

Napolitano, A., Shah, K., Schubert, M.I., Porkess, V., Fone, K.C., Auer, D.P., 2014. In vivo neurometabolic profiling to characterize the effects of social isolation and ketamine-induced NMDA antagonism: a rodent study at 7.0 T. Schizophr Bull 40, 566-574.

Naseribafrouei, A., Hestad, K., Avershina, E., Sekelja, M., Linlokken, A., Wilson, R., Rudi, K., 2014. Correlation between the human fecal microbiota and depression. Neurogastroenterol Motil 26, 1155-1162.

Nestler, E.J., Hyman, S.E., 2010. Animal models of neuropsychiatric disorders. Nature neuroscience $13,1161-1169$.

Nguyen, K.T., Deak, T., Owens, S.M., Kohno, T., Fleshner, M., Watkins, L.R., Maier, S.F., 1998. Exposure to acute stress induces brain interleukin-1beta protein in the rat. J Neurosci 18, 2239-2246.

O'Mahony, S.M., Clarke, G., Dinan, T.G., Cryan, J.F., 2017. Irritable Bowel Syndrome and Stress-Related Psychiatric Co-morbidities: Focus on Early Life Stress. Handb Exp Pharmacol 239, 219-246.

O'Mahony, S.M., Marchesi, J.R., Scully, P., Codling, C., Ceolho, A.M., Quigley, E.M., Cryan, J.F., Dinan, T.G., 2009. Early life stress alters behavior, immunity, and microbiota in rats: implications for irritable bowel syndrome and psychiatric illnesses. Biol Psychiatry 65, 263267.

Ogbonnaya, E.S., Clarke, G., Shanahan, F., Dinan, T.G., Cryan, J.F., O'Leary, O.F., 2015. Adult Hippocampal Neurogenesis Is Regulated by the Microbiome. Biol Psychiatry 78, e7-9. 
Pandey, G.N., Rizavi, H.S., Zhang, H., Ren, X., 2017. Abnormal gene and protein expression of inflammatory cytokines in the postmortem brain of schizophrenia patients. Schizophr Res. Parashar, A., Udayabanu, M., 2016. Gut microbiota regulates key modulators of social behavior. European Neuropsychopharmacology 26, 78-91.

Parker, V., Morinan, A., 1986. The socially-isolated rat as a model for anxiety. Neuropharmacology 25, 663-664.

Perez-Brocal, V., Garcia-Lopez, R., Vazquez-Castellanos, J.F., Nos, P., Beltran, B., Latorre, A., Moya, A., 2013. Study of the viral and microbial communities associated with Crohn's disease: a metagenomic approach. Clin Transl Gastroenterol 4, e36.

Perhach, J.L., Jr., Barry, H., 3rd, 1970. Stress responses of rats to acute body or neck restraint. Physiol Behav 5, 443-448.

Plotsky, P.M., Thrivikraman, K.V., Nemeroff, C.B., Caldji, C., Sharma, S., Meaney, M.J., 2005. Long-term consequences of neonatal rearing on central corticotropin-releasing factor systems in adult male rat offspring. Neuropsychopharmacology 30, 2192-2204.

Pryce, C.R., Ruedi-Bettschen, D., Dettling, A.C., Weston, A., Russig, H., Ferger, B., Feldon, J., 2005. Long-term effects of early-life environmental manipulations in rodents and primates: Potential animal models in depression research. Neurosci Biobehav Rev 29, 649-674.

Pyndt Jorgensen, B., Krych, L., Pedersen, T.B., Plath, N., Redrobe, J.P., Hansen, A.K., Nielsen, D.S., Pedersen, C.S., Larsen, C., Sorensen, D.B., 2015. Investigating the long-term effect of subchronic phencyclidine-treatment on novel object recognition and the association between the gut microbiota and behavior in the animal model of schizophrenia. Physiol Behav 141, 3239. 
Rehman, A., Rausch, P., Wang, J., Skieceviciene, J., Kiudelis, G., Bhagalia, K., Amarapurkar, D., Kupcinskas, L., Schreiber, S., Rosenstiel, P., Baines, J.F., Ott, S., 2016. Geographical patterns of the standing and active human gut microbiome in health and IBD. Gut 65, 238-248.

Robbins, T.W., Jones, G.H., Wilkinson, L.S., 1996. Behavioural and neurochemical effects of early social deprivation in the rat. J Psychopharmacol 10, 39-47.

Rola, R., Raber, J., Rizk, A., Otsuka, S., VandenBerg, S.R., Morhardt, D.R., Fike, J.R., 2004. Radiation-induced impairment of hippocampal neurogenesis is associated with cognitive deficits in young mice. Experimental Neurology 188, 316-330.

Sahakian, B.J., Robbins, T.W., Iversen, S.D., 1977. The effects of isolation rearing on exploration in the rat. Animal Learning \& Behavior 5, 193-198.

Sarkar, A., Lehto, S.M., Harty, S., Dinan, T.G., Cryan, J.F., Burnet, P.W., 2016. Psychobiotics and the manipulation of bacteria-gut-brain signals. Trends Neurosci 39, 763-781.

Savignac, H.M., Couch, Y., Stratford, M., Bannerman, D.M., Tzortzis, G., Anthony, D.C., Burnet, P.W., 2016. Prebiotic administration normalizes lipopolysaccharide (LPS)-induced anxiety and cortical 5-HT2A receptor and IL1-beta levels in male mice. Brain Behav Immun 52, 120-131.

Scaccianoce, S., Del Bianco, P., Paolone, G., Caprioli, D., Modafferi, A.M., Nencini, P., Badiani, A., 2006. Social isolation selectively reduces hippocampal brain-derived neurotrophic factor without altering plasma corticosterone. Behav Brain Res 168, 323-325.

Scarpa, M., Grillo, A., Faggian, D., Ruffolo, C., Bonello, E., D’Incà, R., Scarpa, M., Castagliuolo, I., Angriman, I., 2011. Relationship between mucosa-associated microbiota and inflammatory parameters in the ileal pouch after restorative proctocolectomy for ulcerative colitis. Surgery 150, 56-67. 
Schiavone, S., Sorce, S., Dubois-Dauphin, M., Jaquet, V., Colaianna, M., Zotti, M., Cuomo, V., Trabace, L., Krause, K.H., 2009. Involvement of NOX2 in the development of behavioral and pathologic alterations in isolated rats. Biol Psychiatry 66, 384-392.

Schrijver, N.C., Bahr, N.I., Weiss, I.C., Wurbel, H., 2002. Dissociable effects of isolation rearing and environmental enrichment on exploration, spatial learning and HPA activity in adult rats. Pharmacol Biochem Behav 73, 209-224.

Schubert, M.I., Porkess, M.V., Dashdorj, N., Fone, K.C.F., Auer, D.P., 2009. Effects of social isolation rearing on the limbic brain: a combined behavioral and magnetic resonance imaging volumetry study in rats. Neuroscience 159, 21-30.

Serra, M., Pisu, M.G., Floris, I., Biggio, G., 2005. Social isolation-induced changes in the hypothalamic-pituitary-adrenal axis in the rat. Stress (Amsterdam, Netherlands) 8, 259-264. Severance, E.G., Yolken, R.H., Eaton, W.W., 2016. Autoimmune diseases, gastrointestinal disorders and the microbiome in schizophrenia: more than a gut feeling. Schizophr Res 176, 23-35.

Silva-Gómez, A., 2003. Decreased dendritic spine density on prefrontal cortical and hippocampal pyramidal neurons in postweaning social isolation rats. Brain Research 983, 128136.

Sudo, N., Chida, Y., Aiba, Y., Sonoda, J., Oyama, N., Yu, X.-N., Kubo, C., Koga, Y., 2004. Postnatal microbial colonization programs the hypothalamic-pituitary-adrenal system for stress response in mice. J Physiol 5581, 263-275.

van den Buuse, M., 2010. Modeling the positive symptoms of schizophrenia in genetically modified mice: pharmacology and methodology aspects. Schizophr Bull 36, 246-270.

Watson, D.J.G., King, M.V., Gyertyan, I., Kiss, B., Adham, N., Fone, K.C.F., 2016. The dopamine $D(3)$-preferring $D(2) / D(3)$ dopamine receptor partial agonist, cariprazine, reverses 
behavioural changes in a rat neurodevelopmental model for schizophrenia. Eur Neuropsychopharmacol 26, 208-224.

Weiss, I.C., Pryce, C.R., Jongen-Relo, A.L., Nanz-Bahr, N.I., Feldon, J., 2004. Effect of social isolation on stress-related behavioural and neuroendocrine state in the rat. Behav Brain Res $152,279-295$.

Williams, B.L., Hornig, M., Buie, T., Bauman, M.L., Cho Paik, M., Wick, I., Bennett, A., Jabado, O., Hirschberg, D.L., Lipkin, W.I., 2011. Impaired carbohydrate digestion and transport and mucosal dysbiosis in the intestines of children with autism and gastrointestinal disturbances. PLoS One 6, e24585.

Wongwitdecha, N., Marsden, C.A., 1996. Social isolation increases aggressive behaviour and alters the effects of diazepam in the rat social interaction test. Behavioural Brain Research 75, 27-32.

Woods, S., Clarke, N.N., Layfield, R., Fone, K.C.F., 2012. 5-HT(6) receptor agonists and antagonists enhance learning and memory in a conditioned emotion response paradigm by modulation of cholinergic and glutamatergic mechanisms. Br J Pharmacol 167, 436-449.

Zhang, J.C., Yao, W., Dong, C., Yang, C., Ren, Q., Ma, M., Hashimoto, K., 2017. Blockade of interleukin- 6 receptor in the periphery promotes rapid and sustained antidepressant actions: a possible role of gut-microbiota-brain axis. Transl Psychiatry 7, e1138. 
Figure 1: Protocol diagram showing the time course of experimental housing and behavioural testing across post-natal day age of the Lister hooded rats used in the study.

Figure 2: Comparison of: $(A)$ distance travelled $(\mathrm{cm}),(B)$ average velocity $(\mathrm{cm} / \mathrm{s}),(C)$ time spent (s) in the central and outer zone and (D) number of faecal boli expelled in groupedhoused $(\mathrm{GH})$ and isolation-reared $(\mathrm{SI})$ rats recorded over $15 \mathrm{~min}$ in a circular open field arena. (E) and (F) respectively show the total number of beam breaks and the time course (in consecutive $5 \mathrm{~min}$ epochs) of the same rats recorded in computerised activity boxes over 60min. Data are mean \pm SEM. Figures $A, B, D, E, F *$ indicates difference from $G H$, and in $C *$ indicates difference from the outer zone. ${ }^{* *} \mathrm{P}<0.001,{ }^{*} \mathrm{P}<0.01$ and ${ }^{*} \mathrm{P}<0.05$; for Figures $\mathrm{A}$, $\mathrm{B}, \mathrm{D}$ and $\mathrm{E}$ Student's unpaired $t$-test, and $\mathrm{C}$ and $\mathrm{F}$ Sidak's post-hoc test.

Figure 3: Comparison of the time spent freezing ( $s$, mean $\pm S E M)$ in group-housed $(G H)$ and isolation reared ( $\mathrm{SI}$ ) rats during: $(\mathrm{A})$ 55s following the first and second foot shocks on the conditioning day in the conditioned freezing paradigm $\left({ }^{* *} p<0.001\right.$ from $\mathrm{GH},+++p<0.001$ from SI First Shock, Sidak post-hoc test) or (B) 5 min when rats were returned to the chamber 24 and $48 \mathrm{~h}$ post-conditioning (as indicated, without further foot shock or cue) and at $48 \mathrm{~h}$ post-conditioning with re-exposure to the tone and light conditioning stimulus (48h post-cue). ${ }^{*} p<0.05,{ }^{* *} p<0.01$ from GH, Sidak's post-hoc test following ANOVA.

Figure 4: (A) Representative images of BrdU (red) and NeuN (green) staining; scale bar = $50 \mu \mathrm{m})$ and (B) Number of cells (mean \pm SEM) dual-labelled for BrdU/NeuN in the dentate gyrus of $\mathrm{GH}(\mathrm{n}=11)$ and $\mathrm{SI}(\mathrm{n}=12)$ rats. ${ }^{*} \mathrm{p}<0.05$ Student's unpaired $t$-test.

Fig.5. Comparison of hippocampal (A) IL-1 $\beta$ and (B) IL-6 levels $(n g / m l$, mean \pm SEM, $n=5-6$ each) in group-housed $(\mathrm{GH})$ and rats housed in isolation from weaning (SI) determined 
immediatedly following $1 \mathrm{~h}$ restraint in a perspex tube or continued housing in the home cage, as indicated. ${ }^{*} p<0.05$ from $\mathrm{SI}$ home cage and $\# \mathrm{P}<0.05$ from $\mathrm{GH}$ restrained rats Sidak's multiple comparison test following ANOVA.

Figure 6: Relative abundance of bacteria at phylum level in group-housed (GH, Control) and isolation reared (SI) littermates. The main phyla are Firmicutes.

Figure 7: Relative abundance of bacteria at family level in group-housed (GH, Control) and isolation reared (SI) rats. The main families are Lachnospiraceae and Ruminococcaceae.

Figure 8: Relative abundance of bacteria at genus level in isolation reared (SI) compared with group-housed (GH, Control) rats.

Figure 9: Spearman heatmap focussing on correlation between microbiota and behavioural and cytokine measures found to be significantly different as a consequence of housing during development. Top x-axis columns: Conditioned Freezing Response (CFR) task, hippocampal (Hipp) cytokines, and open field (OF) left to right, respectively. Y-axis groups taxa by phylogeny (right y-axis) and corresponding taxonomic names (left y-axis). Scale (right legend) indicates level of positive (red) or negative (blue) correlation, asterisk indicates significance $\left({ }^{*} \mathrm{P}<0.05,{ }^{* *} \mathrm{P}<0.01\right.$ and $\left.{ }^{* * *} \mathrm{P}<0.001\right)$. 
Figure 1

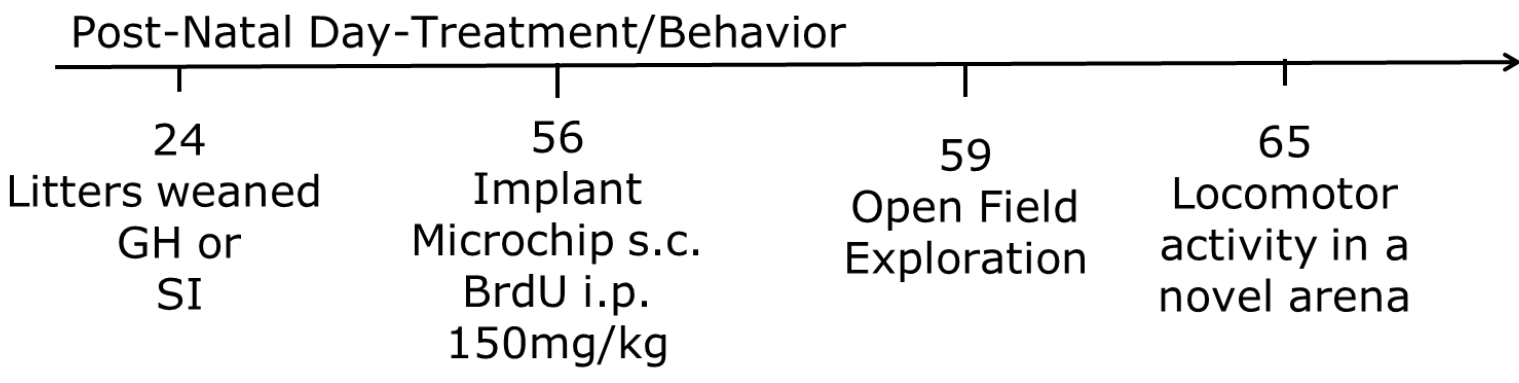

Post-Natal Day-Behavior

$66 \quad 73 \quad 79 / 80 / 81 \quad 86 / 87$

Novel object Elevated plus Conditioned Restraint Stress and discrimination maze Freezing Sample Collection

Response 
Figure 2

A

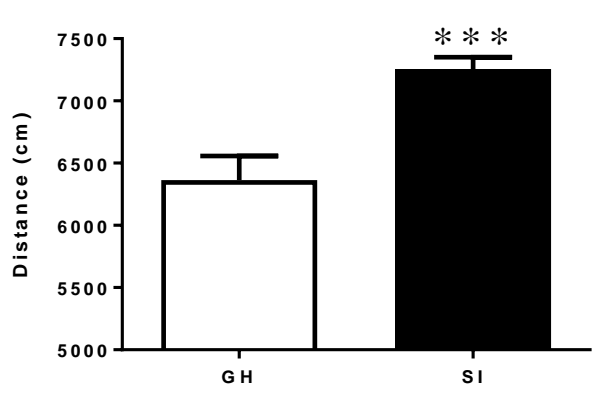

C

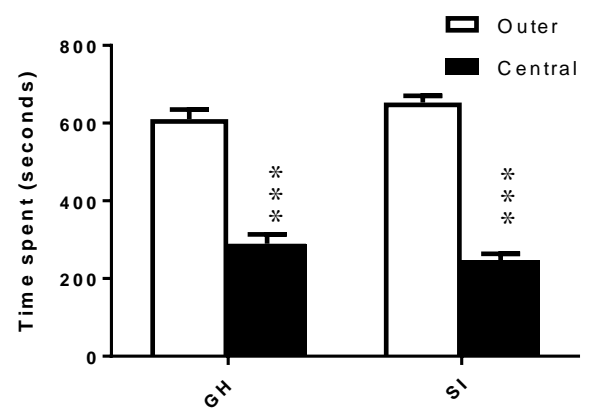

E

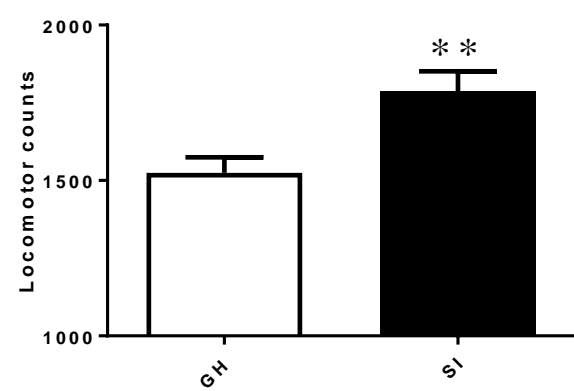

B
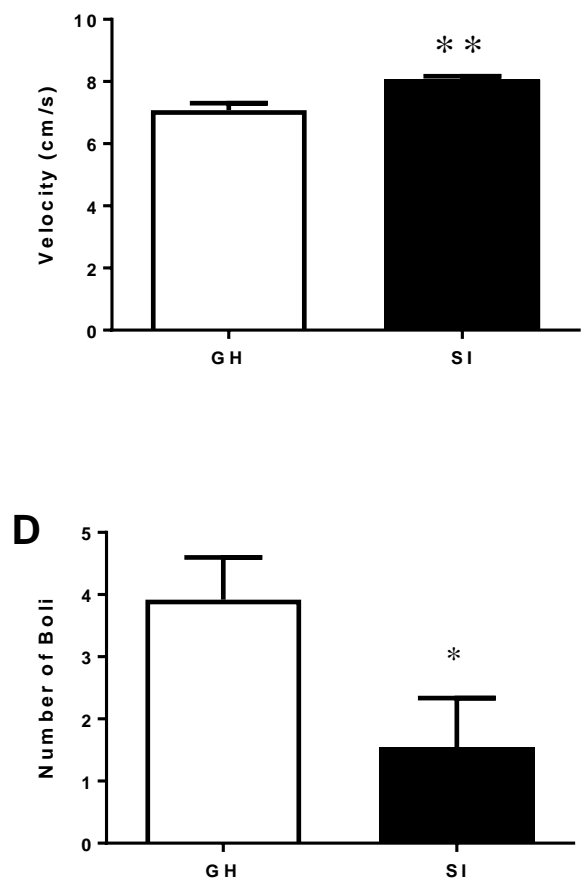

F

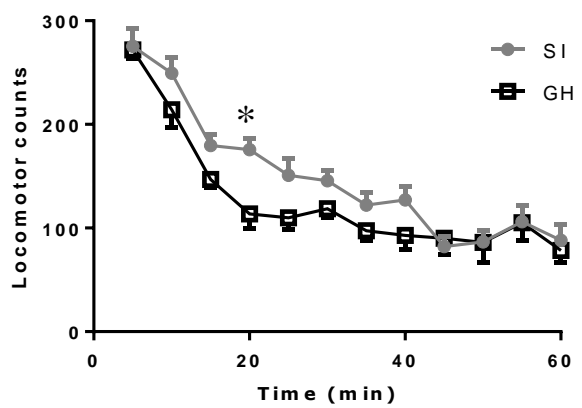


Figure 3

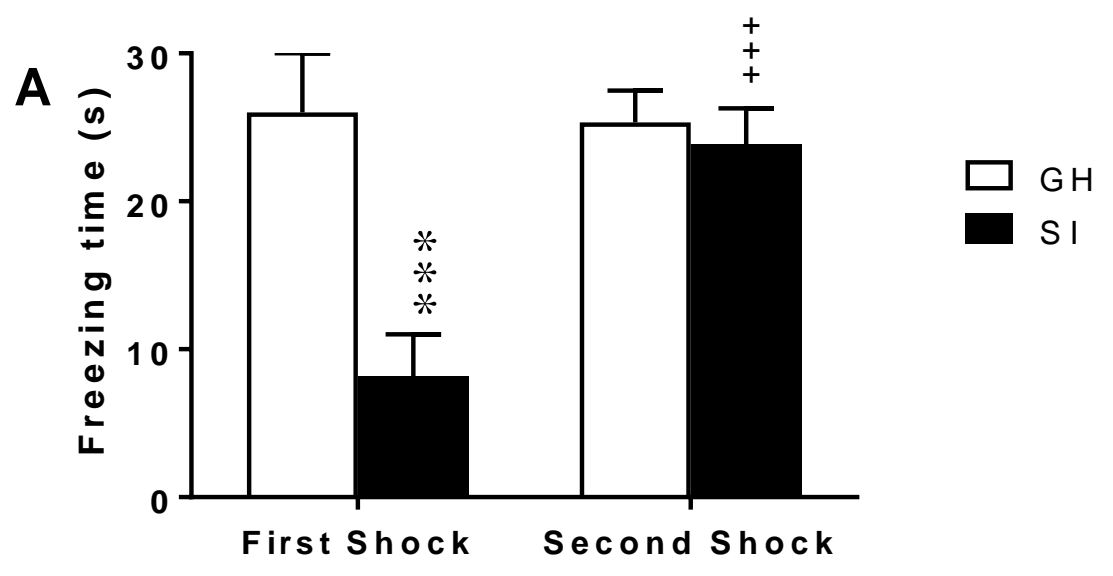

B

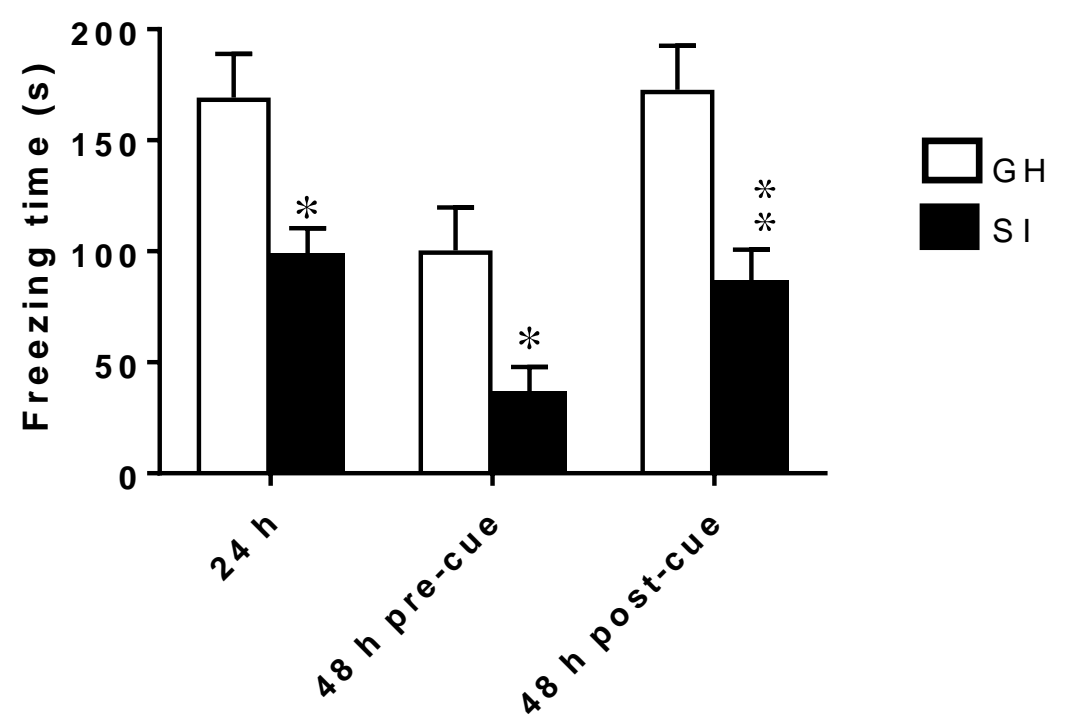


Figure 4
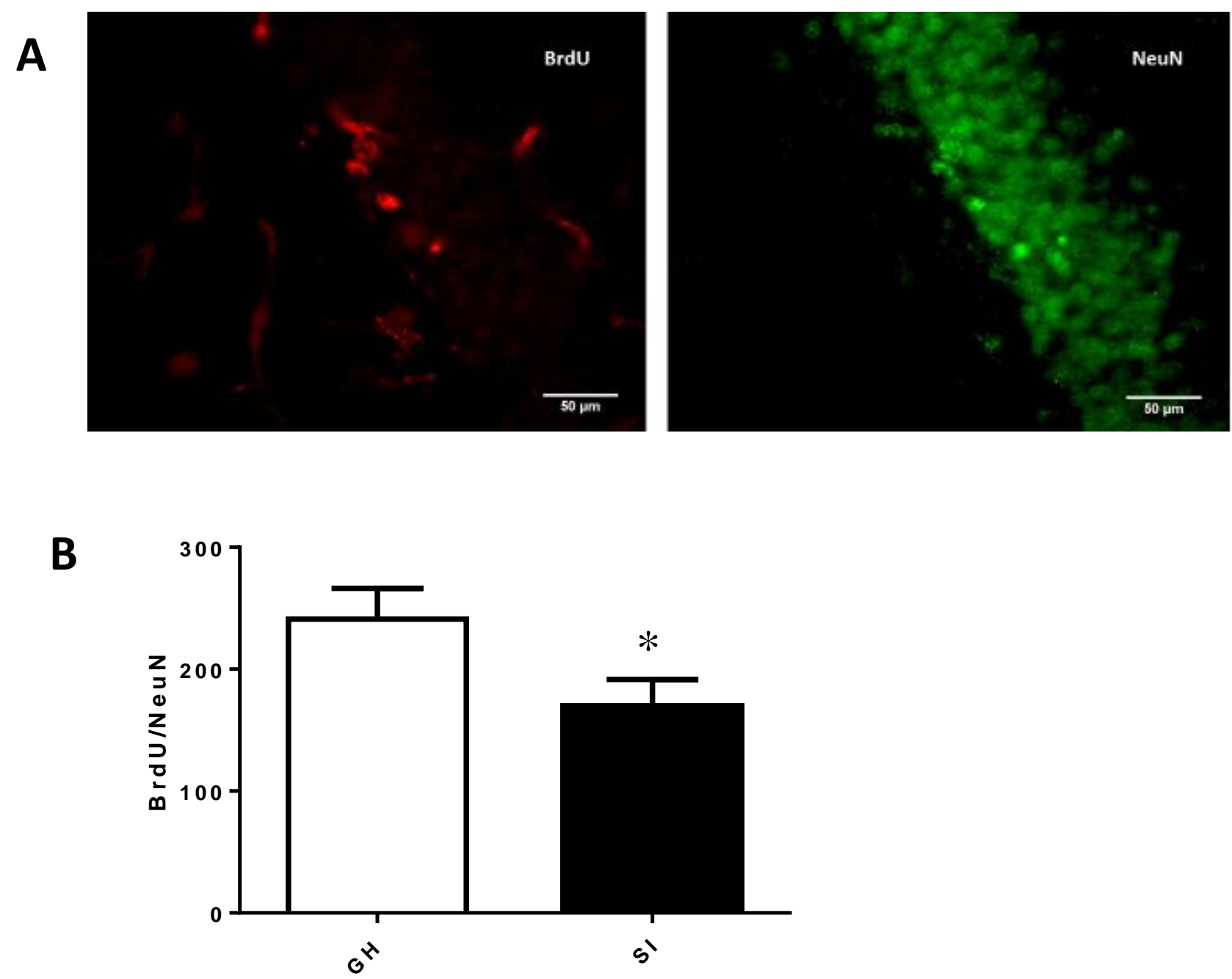

Housing 
Figure 5
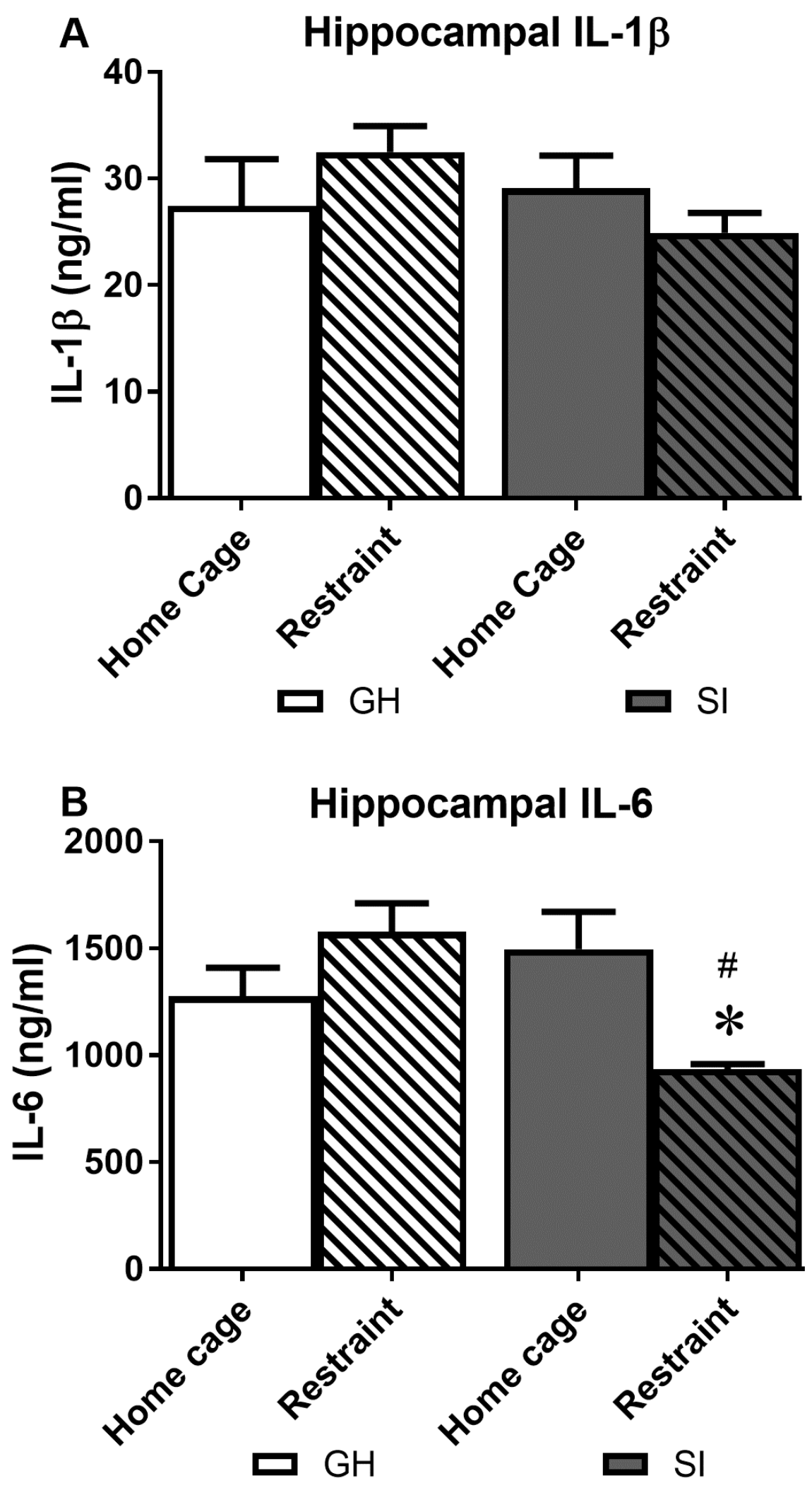
Figure 6

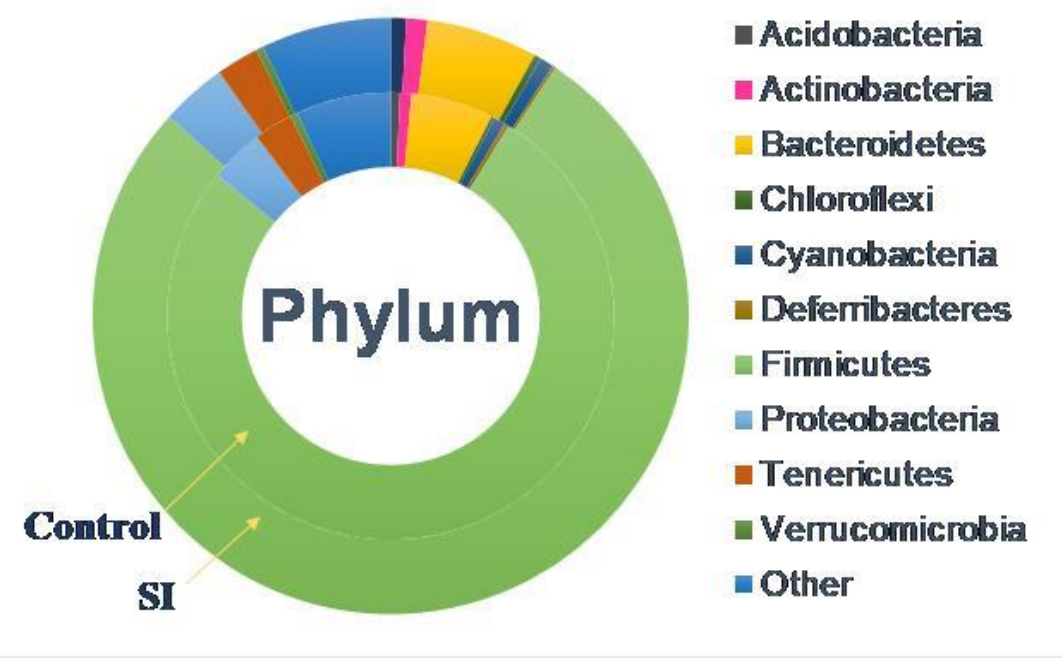


Figure 7

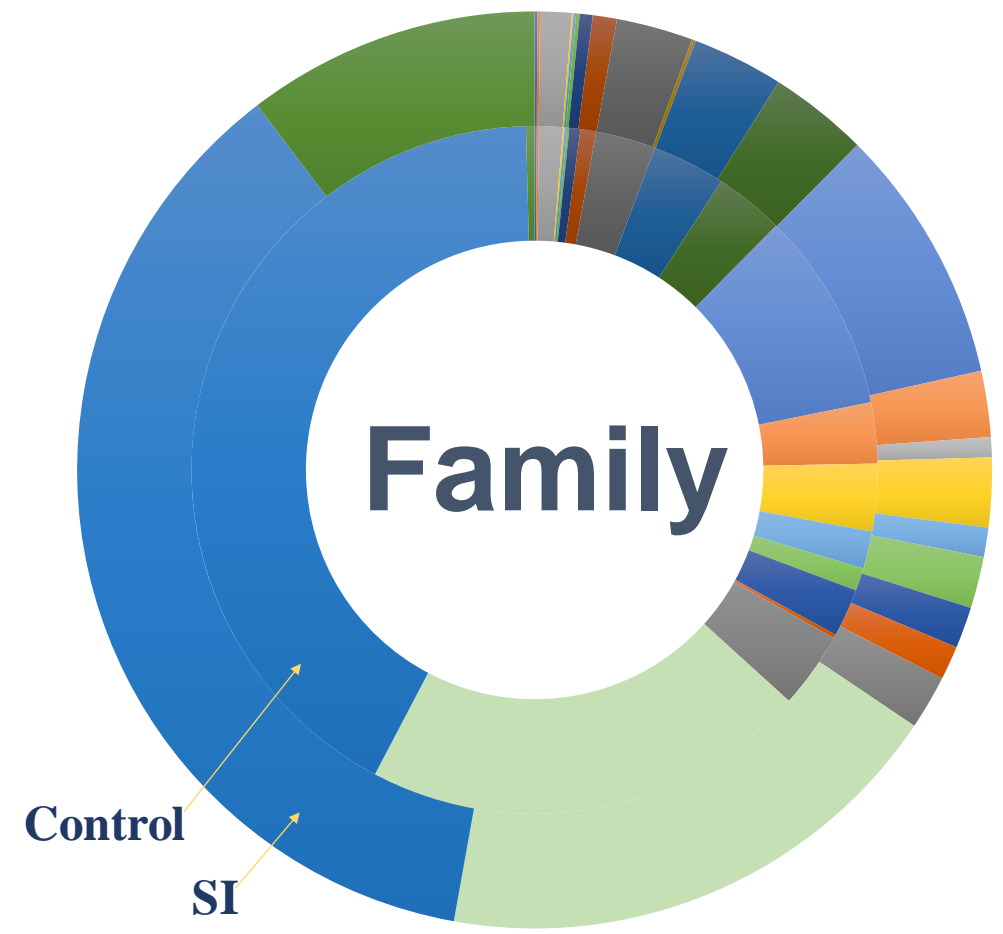

- Family XIII

- Bifidobacteriaceae

- Rikenellaceae

- Christensenellaceae

- ratAN060301C

- Alcaligenaceae

- Peptococcaceae

- Porphyromonadaceae

- Bacteroidaceae

- Bacillaceae

- Erysipelotrichaceae

- Bacteroidales S24-7

- Prevotellaceae

- Lactobacillaceae

- Mitochondria

- Clostridiales vadinBB60

- Clostridiaceae

- Verrucomicrobiaceae

- Deferribacteraceae

- Anaeroplasmataceae

- Peptostreptococcaceae

- Ruminococcaceae

- Lachnospiraceae

- Others 
Figure 8

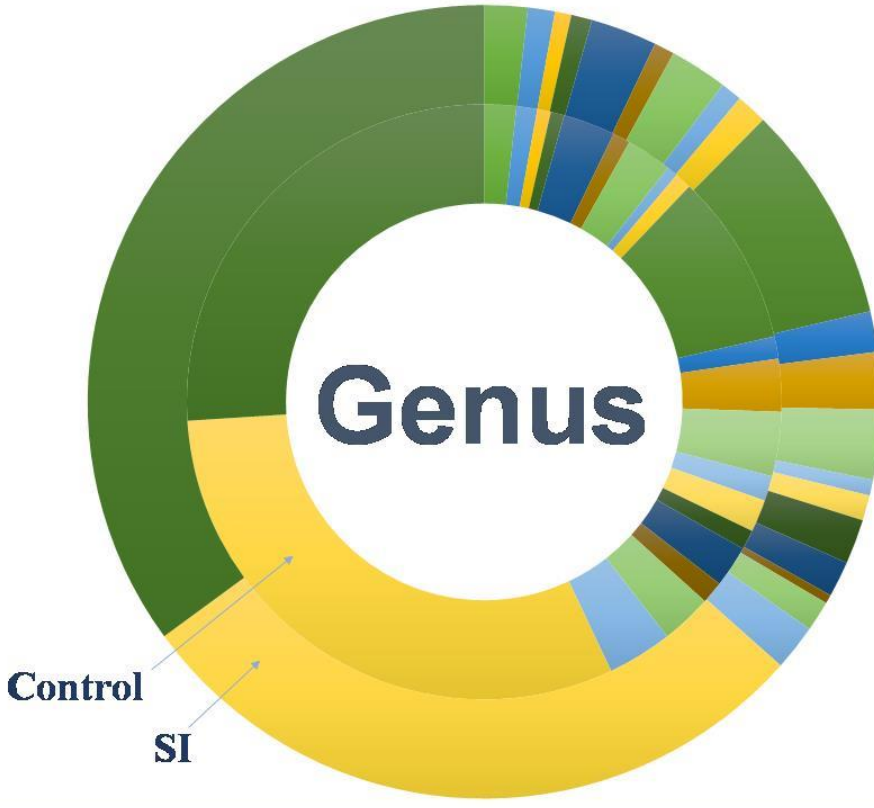

\author{
- Ruminococcus \\ - Alistipes \\ Lachnospiraceae Incertae Sedis \\ - Parabacteroides \\ - Bacteroides \\ - Turicibacter \\ - Asteroleplasma \\ - Eubacterium coprostanoligenes \\ - Oscillibacter \\ - Prevotellaceae NK3B31 \\ - Ruminococcaceae UCG-014 \\ - Lactobacillus \\ - Ruminiclostridium \\ - Lachnospiraceae UCG-001 \\ - Clostridium sensu stricto \\ - Akkermansia \\ - Mucispirillum \\ - Eubacterium ruminantium \\ - Ruminococcaceae UCG-003 \\ - Peptoclostridium \\ - Lachnospiraceae NK4A136 \\ - Others
}


Figure 9

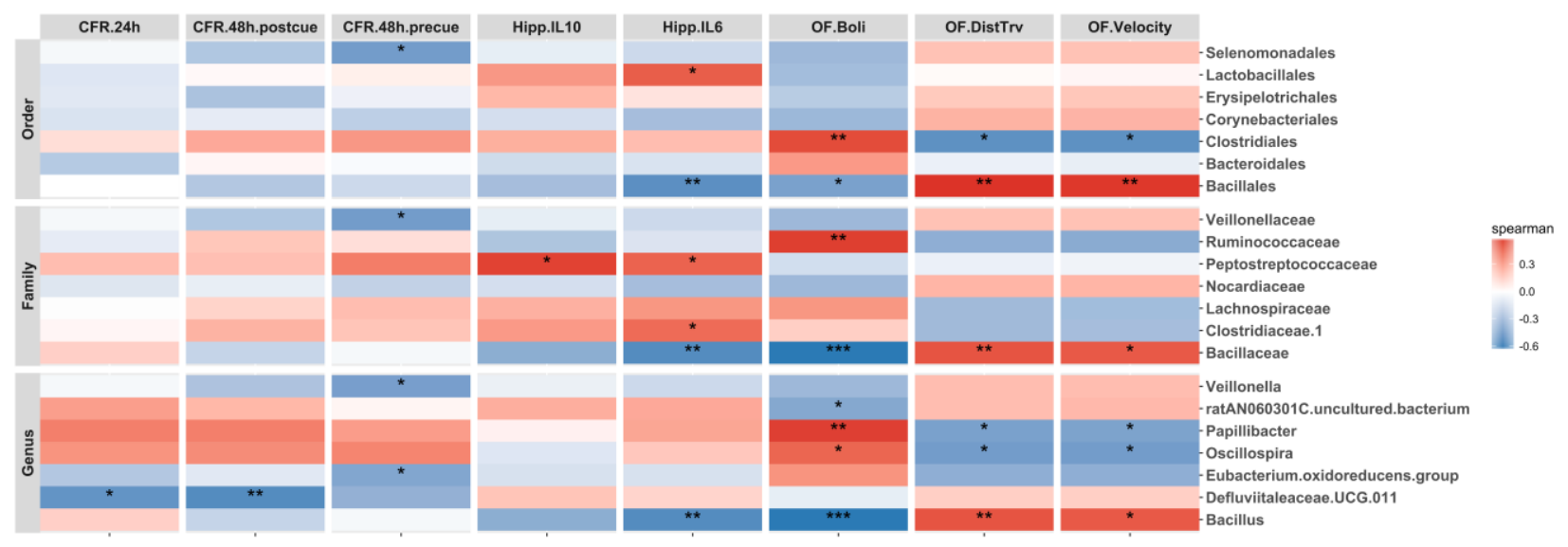


Table 1: Lack of effect of housing condition $(\mathrm{GH}=$ group and $\mathrm{SI}=$ individual housing during rearing) on time (s, mean \pm SEM) spent exploring two identical objects (front and back) in the first familiarisation (T1), the novel and familiar object in the second (T2) choice trial, and the choice trial discrimination ratio (novel/novel + familiar times) during the novel object discrimination task.

Table 2: Cytokine levels (mean $\pm \mathrm{SEM}, \mathrm{ng} / \mathrm{ml}$ ) in hippocampus and prefrontal cortex (PFC) of $\mathrm{GH}$ and SI rats. Any sample whose cytokine value was outside the linear portion of the curve was omitted. ${ }^{*} \mathrm{p}<0.05$ Unpaired Student's $t$-test from hippocampal GH control.

Table 3: Levels of mTOR, phosphor-mTOR and their ratio (mean \pm SEM) in the hippocampus and prefrontal cortex (PFC) of rats housed in social groups (GH) or isolation (SI), measured post-mortem using ELISA. No significant differences were found between housing groups for mTOR and phospho-mTOR in either the hippocampus and PFC but there was a significant increase in the ratio of phospho-mTOR to total mTOR in the PFC $\left({ }^{*} p<0.05\right.$, Student's unpaired $t$-test).

Table 4: Summary of the significant changes in bacterial composition in isolation reared (SI) relative to group-housed $(\mathrm{GH})$ rats. Taxa highlighted in yellow indicates significance $(p<0.05)$, arrows indicate increases or decrease in SI relative to GH rats. 
Table 1

\begin{tabular}{|l|c|c|}
\hline Object type and Trial & GH & SI \\
\hline Front Object T1 & $19.9 \pm 2.4$ & $23.2 \pm 2.6$ \\
\hline Back Object T1 & $24.5 \pm 3.5$ & $23.1 \pm 2.3$ \\
\hline Familiar Object T2 & $17.3 \pm 3.2$ & $13.4 \pm 2.1$ \\
\hline Novel Object T2 & $21.5 \pm 1.7$ & $15.8 \pm 3.4$ \\
\hline Discrimination Ratio T2 & $0.6 \pm 0.1$ & $0.5 \pm 0.1$ \\
& & \\
\hline
\end{tabular}


Table 2

\begin{tabular}{|c|c|c|c|c|}
\hline Cytokine & $\begin{array}{c}\text { GH } \\
\text { Hippocampus }\end{array}$ & $\begin{array}{c}\text { SI } \\
\text { Hippocampus }\end{array}$ & $\begin{array}{l}\text { GH } \\
\text { PFC }\end{array}$ & $\begin{array}{l}\text { SI } \\
\text { PFC }\end{array}$ \\
\hline IL-1 $\beta$ & $\begin{array}{c}30.20 \pm 2.38 \\
(n=11)\end{array}$ & $\begin{array}{c}25.49 \pm 1.12 \\
(n=11)\end{array}$ & $\begin{array}{c}32.14 \pm 2.84 \\
(n=11)\end{array}$ & $\begin{array}{c}32.64 \pm 1.73 \\
(n=12)\end{array}$ \\
\hline IL-6 & $\begin{array}{c}1441 \pm 101.3 \\
(n=11)\end{array}$ & $\begin{array}{c}1137 \pm 98.6 \\
(n=11) *\end{array}$ & $\begin{array}{c}491.9 \pm 77.7 \\
(n=10)\end{array}$ & $\begin{array}{c}504.6 \pm 30.8 \\
(n=11)\end{array}$ \\
\hline IL-10 & $\begin{array}{c}9.58 \pm 1.98 \\
(n=10)\end{array}$ & $\begin{array}{c}4.84 \pm 0.75 \\
(n=8) *\end{array}$ & $\begin{array}{c}14.97 \pm 3.66 \\
(n=9)\end{array}$ & $\begin{array}{c}7.67 \pm 1.67 \\
(n=11)\end{array}$ \\
\hline TNF $\alpha$ & $\begin{array}{c}0.31 \pm 0.05 \\
(n=10)\end{array}$ & $\begin{array}{c}0.21 \pm 0.04 \\
(n=8)\end{array}$ & $\begin{array}{c}0.27 \pm 0.05 \\
(n=6)\end{array}$ & $\begin{array}{c}0.18 \pm 0.02 \\
(n=7)\end{array}$ \\
\hline
\end{tabular}


Table 3

\begin{tabular}{|l|c|c|c|c|}
\hline & GH & SI & GH & SI \\
& Hippocampus & Hippocampus & PFC & PFC \\
\hline mTOR & $3886 \pm 161$ & $3255 \pm 281$ & $2409 \pm 114$ & $2071 \pm 129$ \\
& $(n=10)$ & $(n=12)$ & $(n=10)$ & $(n=12)$ \\
\hline Phospho- & $10.60 \pm 0.74$ & $9.73 \pm 1.36$ & $5.58 \pm 0.82$ & $7.79 \pm 0.87$ \\
mTOR & $(n=10)$ & $(n=12)$ & $(n=9)$ & $(n=11)$ \\
& & & & $(n=11) *$ \\
\hline Ratio & $0.0028 \pm 0.0002$ & $0.0029 \pm 0.0004$ & $0.0024 \pm 0.0003$ & $0.0036 \pm 0.0003$ \\
& $(n=11)$ & $(n=12)$ & $(n=9)$ & \\
\hline
\end{tabular}


Table 4

\begin{tabular}{|llllll|}
\hline Kingdom & Phylum & Class & Order & Family & Genus \\
\hline Bacteria & $\uparrow$ Actinobacteria & Actinobacteria & $\uparrow$ Corynebacteriales & $\uparrow$ Nocardiaceae & $\uparrow$ Rhodococcus \\
Bacteria & Bacteroidetes & Bacteroidia & Bacteroidales & Prevotellaceae & $\uparrow$ Prevotellaceae UCG-o01 \\
Bacteria & Bacteroidetes & Bacteroidia & Bacteroidales & ratAN060301C & $\uparrow$ uncu/tured bacterium \\
Bacteria & Firmicutes & Bacilli & $\uparrow$ Bacillales & $\uparrow$ Bacillaceae & $\uparrow$ Bacillus \\
Bacteria & Firmicutes & $\downarrow$ Clostridia & $\downarrow$ Clostridiales & $\downarrow$ Clostridiaceae 1 & Clostridium sensu stricto 1 \\
Bacteria & Firmicutes & $\downarrow$ Clostridia & $\downarrow$ Clostridiales & Defluviitaleaceae & $\uparrow$ Defluviitaleaceae UCG-011 \\
Bacteria & Firmicutes & $\downarrow$ Clostridia & $\downarrow$ Clostridiales & Lachnospiraceae & $\uparrow$ Eubacterium oxidoreducens group \\
Bacteria & Firmicutes & $\downarrow$ Clostridia & $\downarrow$ Clostridiales & Lachnospiraceae & $\uparrow$ Marvinbryantia \\
Bacteria & Firmicutes & $\downarrow$ Clostridia & $\downarrow$ Clostridiales & Lachnospiraceae & $\downarrow$ Lachnospiraceae UCG-o09 \\
Bacteria & Firmicutes & $\downarrow$ Clostridia & $\downarrow$ Clostridiales & Ruminococcaceae & $\downarrow$ Oscillospira \\
Bacteria & Firmicutes & $\downarrow$ Clostridia & $\downarrow$ Clostridiales & Ruminococcaceae & $\downarrow$ Papillibacter \\
Bacteria & Firmicutes & $\downarrow$ Clostridia & $\downarrow$ Clostridiales & $\downarrow$ Peptostreptococcaceae & Peptoclostridium \\
Bacteria & Firmicutes & $\uparrow$ Negativicutes & $\uparrow$ Selenomonadales & $\uparrow$ Veillonellaceae & $\uparrow$ Veillonella \\
\hline
\end{tabular}

$\downarrow$ Decrease, $\uparrow$ Increase in SI relative to $\mathrm{GH}$

Significant at taxanomic level $(p<0.05)$ 